\title{
Galectin-1-driven T cell exclusion in the tumor endothelium promotes immunotherapy resistance
}

\author{
Dhanya K. Nambiar, ${ }^{1}$ Todd Aguilera, ${ }^{2}$ Hongbin Cao, ${ }^{1}$ Shirley Kwok, ${ }^{3}$ Christina Kong, ${ }^{3}$ Joshua Bloomstein, ${ }^{1}$ Zemin Wang, ${ }^{4}$ \\ Vangipuram S. Rangan, ${ }^{4}$ Dadi Jiang, ${ }^{5}$ Rie von Eyben, ${ }^{1}$ Rachel Liang, ${ }^{1}$ Sonya Agarwal, ${ }^{1}$ A. Dimitrios Colevas, ${ }^{6}$ Alan Korman, ${ }^{4}$ \\ Clint T. Allen, ${ }^{7}$ Ravindra Uppaluri, ${ }^{8}$ Albert C. Koong, ${ }^{5}$ Amato Giaccia, ${ }^{1}$ and Quynh Thu Le ${ }^{1}$

\begin{abstract}
'Department of Radiation Oncology, Stanford University School of Medicine, Stanford, California, USA. ${ }^{2}$ Department of Radiation Oncology, University of Texas Southwestern, Dallas, Texas, USA. ${ }^{3}$ Department of Pathology, Stanford University School of Medicine, Stanford, California, USA. ${ }^{4}$ Biologics Discovery California, Bristol-Myers Squibb, Redwood City, California, USA. ${ }^{5}$ Department of Radiation Oncology,

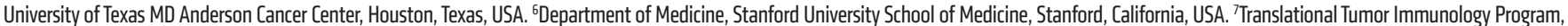
National Institute on Deafness and Other Communication Disorders (NIDCD), Bethesda, Maryland, USA. ${ }^{8}$ Department of Surgery - Otolaryngology, Brigham and Women's Hospital and Dana-Farber Cancer Institute, Boston, Massachusetts, USA.
\end{abstract}

\begin{abstract}
Immune checkpoint inhibitors (ICls), although promising, have variable benefit in head and neck cancer (HNC). We noted that tumor galectin-1 (Gal1) levels were inversely correlated with treatment response and survival in patients with HNC who were treated with ICls. Using multiple HNC mouse models, we show that tumor-secreted Gal1 mediates immune evasion by preventing T cell migration into the tumor. Mechanistically, Gal1 reprograms the tumor endothelium to upregulate cellsurface programmed death ligand 1 (PD-L1) and galectin-9. Using genetic and pharmacological approaches, we show that Gal1 blockade increases intratumoral T cell infiltration, leading to a better response to anti-PD1 therapy with or without radiotherapy. Our study reveals the function of Gal1 in transforming the tumor endothelium into an immune-suppressive barrier and that its inhibition synergizes with ICls.
\end{abstract}

\section{Introduction}

Immunosuppression is an accepted hallmark of tumor progression, and targeting immune-suppressive pathways has led to enhanced patient survival in multiple cancers, including head and neck cancer (HNC) $(1,2)$. Unfortunately, many cancers show a poor response to immune checkpoint inhibitors (ICIs), because T cells, which are the final common effector for most immune-activating strategies, are excluded by hostile tumor microenvironments (1). Studies analyzing tumor biopsies across different cancer types show that $\mathrm{T}$ cell infiltration into the tumor is an important biomarker for predicting ICI responses (3-5). In fact, T cell exclusion remains one of the most salient characteristics of many tumors $(6$, 7). Tumors achieve immunosuppression using multiple pathways involving intrinsic cell behavior as well as extrinsic factors (6). The tumor stroma, especially the tumor endothelium, plays a critical role in shaping the immune landscape within the tumor $(8,9)$. Recent studies suggest that tumor endothelium can switch roles from being a passive facilitator of $\mathrm{T}$ cell infiltration to an active inhibitor, thereby heightening immunosuppression (10-12).

\section{Related Commentary: p. 5089}

Conflict of interest: AK, ZW, and VR are employees and stockholders of Bristol-Myers Squibb, which makes anti-galectin-1 and anti-PD1 antibodies. Copyright: ( 2019, American Society for Clinical Investigation. Submitted: March 25, 2019; Accepted: September 12, 2019; Published: November 11, 2019 Reference information: J Clin Invest. 2019;129(12):5553-5567. https://doi.org/10.1172/JCl129025.
Tumors secrete various growth factors and cytokines to shape an immunosuppressive environment. Galectin-1 (Gal1), a carbohydrate-binding protein, is highly overexpressed and secreted into the surrounding milieu by solid cancers including lung, melanoma, breast, and HNC (13-15). Gal1 has been studied in the context of tumor angiogenesis and immune modulation (16-18). Previously, our group found a strong inverse correlation between the hypoxiarelated marker Gal1 and CD3 staining in patients with HNC, providing evidence of how hypoxia can help tumors evade immune surveillance (14). Most studies evaluating the immune effects of Gal1 in cancer have focused on its role in causing T cell apoptosis (19). However, the relevance of Gal1 in the tumor microenvironment has been questioned, because physiological concentrations of Gal1 are unlikely to reach the concentrations necessary to induce apoptosis $(20,21)$. Because Gal1 shows a strong inverse correlation with $\mathrm{T}$ cell levels and a direct relationship with tumor progression in HNCs, we decided to evaluate whether Gal1 could be a significant player in causing $\mathrm{T}$ cell exclusion and immunotherapy resistance. Here, in a cohort of patients with recurrent/metastatic HNC, we report that patients with high tumoral or stromal Gal1 expression had worse overall survival when treated with ICIs than did those with low expression. Our preclinical studies demonstrated that Gal1 actively remodeled the tumor endothelium to suppress $\mathrm{T}$ cell infiltration, leading to T cell exclusion from the tumor microenvironment. We demonstrate that sustained Gal1 release from the tumor was sufficient to activate STAT signaling pathway in endothelial cells (ECs). These cells, in turn, upregulated immune checkpoint ligands, which then mitigated intratumoral $\mathrm{T}$ cell infiltration. 

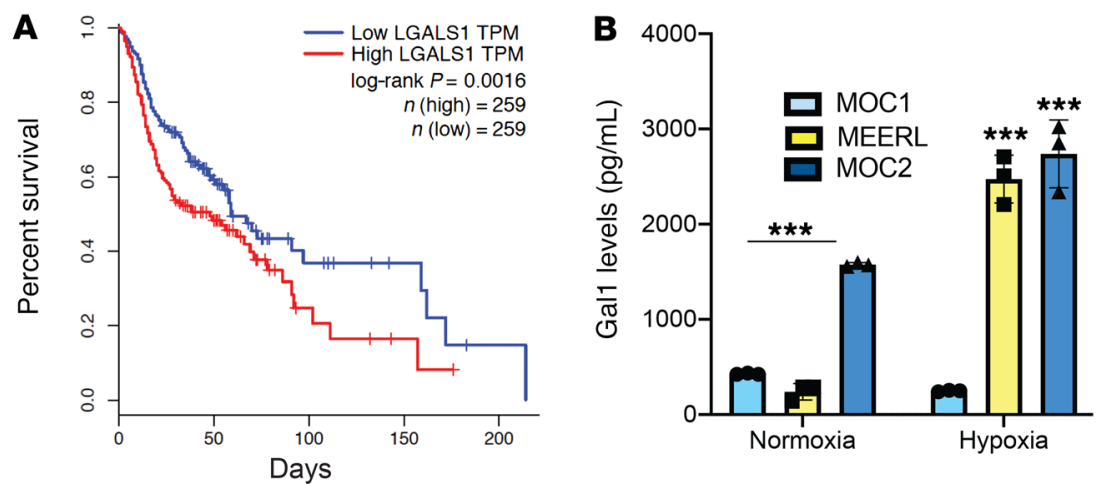

C
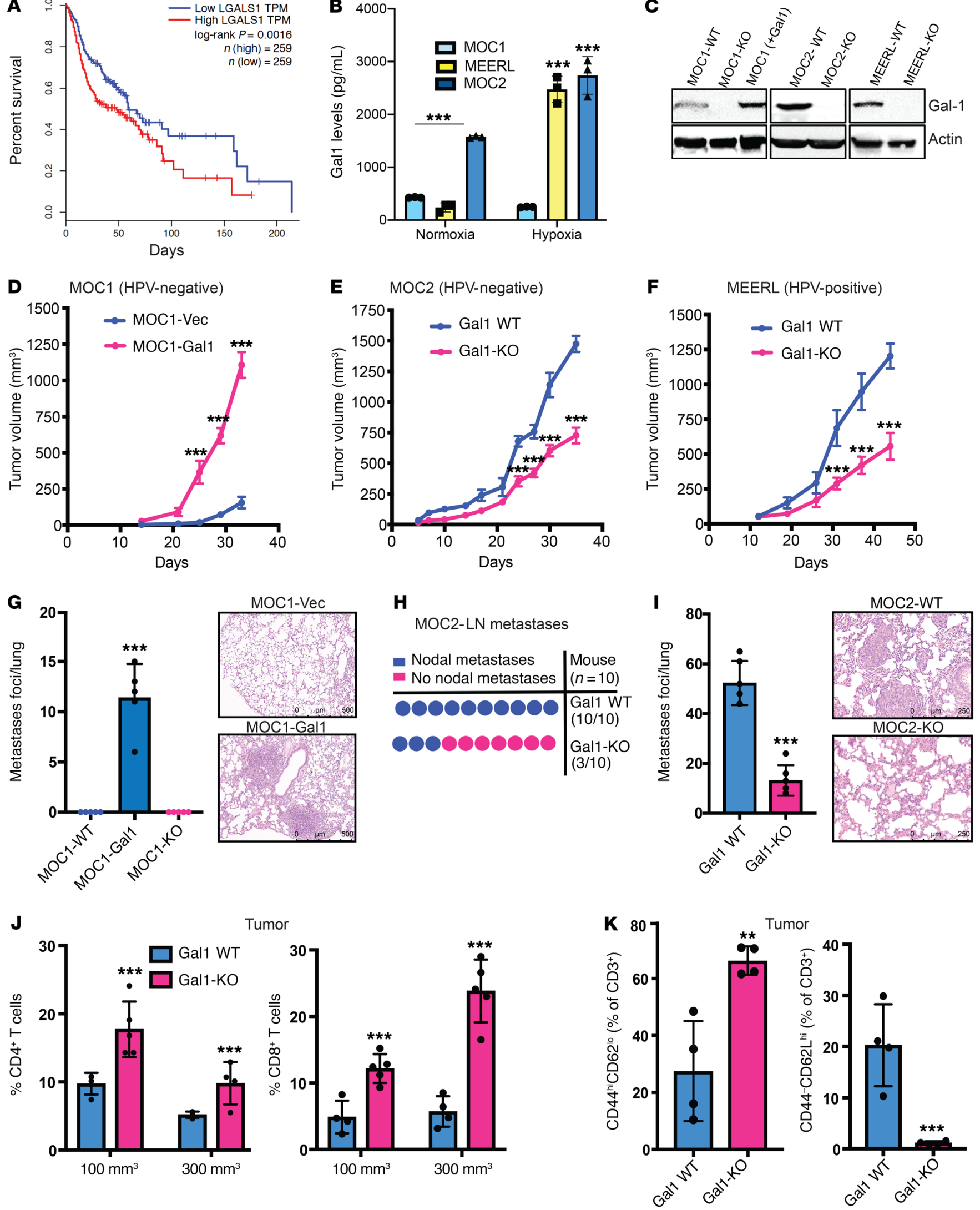
Figure 1. Gal1 promotes tumor growth and metastases in a HNC model by causing immune suppression. (A) Kaplan-Meier analysis of overall survival of patients with HNSCC according to Gal1 gene expression ( $n=$ 518 patients, TCGA data set). $P=0.0016$. (B) ELISA results for secreted levels of Gal1 in murine HNSCC cells (MOC1, MEERL, and MOC2) after 24 hours of normoxia or hypoxia $\left(0.5 \% \mathrm{O}_{2}\right)$. (C) Immunoblots show Gal1 deletion with CRISPR/Cas9 in MOC1, MOC2, and MEERL cells and stable lentiviral overexpression of Gal1 in MOC1 (MOC1 + Gal1) cells. (D) Tumor growth curves for C57BL/ 6 mice subcutaneously implanted with $1 \times 10^{6}$ MOC1 vector control cells (MOC1-Vec) or MOC1 Gal1-overexpressing cells

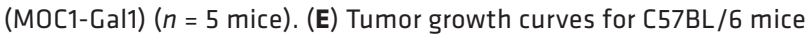
subcutaneously implanted with $2.5 \times 10^{5} \mathrm{MOC2}$ Gal1 WT or Gal1-KO cells ( $n=5$ mice). (F) Tumor growth curves for C57BL/ 6 mice subcutaneously implanted with $1 \times 10^{6}$ MEERL Gal1 WT or Gal1-KO cells ( $n=5$ mice/ group). (G) Quantification of lung metastases foci after subcutaneous implantation of each cell line. The number of nodules per lung area was quantified by $\mathrm{H} \& \mathrm{E}$ staining (scale bars: $500 \mu \mathrm{m}$ ). In the graph, each dot represents 1 mouse, and the bar indicates the mean. (H) Quantification of LN metastases in mice bearing either MOC2 Gal1 WT or Gal1-KO tumors. (I) Quantification and representative histologic images of metastatic foci in lungs after subcutaneous implantation of MOC2 Gal1 WT or Gal1-KO cells, measured at comparable primary tumor sizes. Scale bars: $250 \mu \mathrm{m}$. (J) Quantification of CD4 ${ }^{+}$and CD8 ${ }^{+} \mathrm{T}$ cells in MOC2 Gal1 WT and Gal1-KO tumors at sizes of approximately $100 \mathrm{~mm}^{3}$ and $300 \mathrm{~mm}^{3}$, after enzymatic dissociation and flow cytometric analyses. (K) Flow cytometric analyses of CD44 and CD62L markers on $\mathrm{CD}^{+} \mathrm{T}$ cells from MOC2 Gal1 WT and Gal1-KO tumors. ${ }^{* *} P<0.01$ and ${ }^{* *} P<$ 0.001 . Overall survival was summarized using Kaplan-Meier curves, and groups were compared using log-rank tests $(\mathbf{A})$; repeated-measures ANOVA was used for tumor growth measurement over time (D-F); and a 2-tailed Student's $t$ test was used for comparisons of single treatment with the control (B, G, and I-K).

Targeting Gal1 therapeutically using a Gal1-specific antibody converted a $\mathrm{T}$ cell-desolate tumor into one enriched for $\mathrm{T}$ cells that became responsive to anti-PD1 therapy and radiation therapy. This work provides insights into how tumor-secreted Gal1 conditions the tumor endothelium to enhance $\mathrm{T}$ cell exclusion and promotes systemic tolerance in HNC.

\section{Results}

Gal1 contributes to HNC progression by establishing an immune-suppressive microenvironment. We previously showed that Gal1 secretion was significantly induced in tumors under hypoxic conditions and that its gene expression levels correlated with worse overall survival in a cohort of patients with HNC (14). We extended this finding to The Cancer Genome Atlas (TCGA) data with a larger number of HNC patients $(n=518)$ and confirmed that Gal1 mRNA levels were inversely correlated with overall survival in this group $(P=0.0016)$ (Figure 1A). We also compared the gene expression levels of 3 major galectin family members (Gal1, -3 , and -9) in the tumor using the above HNC TCGA cohort with levels in healthy tissues (GTEx cohort, $n=44$ ) and found that only Gal1 was significantly overexpressed in tumor samples (Supplemental Figure 1A; supplemental material available online with this article; https:// doi.org/10.1172/JCI129025DS1). CIBERSORT analyses revealed a significant inverse relationship between Gal1 expression and $\mathrm{CD} 4^{+}$memory resting $\mathrm{T}$ cells and $\mathrm{CD} 4^{+}$follicular $\mathrm{T}$ cells, but not $\mathrm{CD}^{+} \mathrm{T}$ cells (Supplemental Figure 1B). This finding further highlights the role of Gal1 in tumor progression in HNC and its relationship to intratumoral $\mathrm{T}$ cell infiltration.
For preclinical studies, we used both $\mathrm{HPV}^{-}$(MOC1 and MOC2) and $\mathrm{HPV}^{+}$(MEERL) mouse syngeneic HNC models, both of which show high fidelity to human disease in terms of biologic behavior and genomic landscape $(22,23)$. MOC1 and MOC2 cells show different levels of aggressiveness with regard to tumor growth and metastasis in mice. The highly metastatic and aggressive MOC2 cells secreted high basal levels of Gal1 under normoxia (21\%), which was further enhanced in hypoxia (0.5\%). The slow-growing, nonmetastatic MOC1 cells secreted low levels of Gal1 under both conditions. Moderately aggressive MEERL cells showed low Gal1 secretion under normoxia but elevated secretion under hypoxia (Figure 1B). These tumor models thus replicated the prior findings in other cancer types that correlated Gal1 levels with tumor aggressiveness (24). We next induced overexpression of Gal1 in MOC1 cells and knocked out Gal1 using CRISPR/Cas9 gene targeting in all 3 cell lines (Figure 1C). Gal1 overexpression in MOC1 cells led to enhanced tumor growth and spontaneous lung metastases in a subcutaneous C57BL/6 tumor model (Figure 1, D and G). In contrast, Gal1 deletion resulted in a significant reduction $(-50 \%, P<0.001)$ in primary growth of MOC2 and MEERL tumors (Figure 1, E and F). Gal1-depleted MOC2 tumor-bearing mice exhibited substantially fewer spontaneous nodal and lung metastases, even when the primary tumors were size matched with their respective parental controls (Figure $1, \mathrm{H}$ and I). Since the tumor microenvironment is highly dependent on the tumor growth site, we also verified these effects with an orthotopic buccal cavity model using MOC2 cells in C57/BL6 mice. We observed a similar decrease in tumor growth in Gal1null compared with WT tumors, as previously observed in the subcutaneous models (Supplemental Figure 1C). Flow cytometric analysis of dissociated MOC2 tumors, at small sizes $\left(100 \mathrm{~mm}^{3}\right)$, showed an approximately 2 -fold increase in $\mathrm{CD} 4^{+} \mathrm{T}$ cells and a 3-fold increase in $\mathrm{CD}^{+} \mathrm{T}$ cells in the Gal1-KO versus WT tumors (Figure 1J). In larger tumors $\left(300 \mathrm{~mm}^{3}\right)$, we found that the CD8 ${ }^{+}$ $\mathrm{T}$ cell population increased significantly from $5.7 \%$ in Gal1 WT tumors to $23.7 \%$ in Gal1-KO tumors (Figure 1J). We made similar observations in the MOC1 model, in which Gal1 overexpression led to an overall reduction in $\mathrm{CD} 45^{+}$infiltrating leukocytes (20.2\% in vector control vs. $4.2 \%$ in Gal1-overexpressing tumors, Supplemental Figure 1D) and $\mathrm{CD}^{+} \mathrm{T}$ cells (5.9\% in vector control vs. $0.5 \%$ in Gal1-overexpressing tumors, Supplemental Figure $1 \mathrm{E})$. Detailed analysis of infiltrated T cells in the MOC2 model revealed that most of the $\mathrm{T}$ cells in Gal1-KO tumors were effector memory cells $\left(\mathrm{CD} 44^{\mathrm{hi}} \mathrm{CD} 62 \mathrm{~L}^{\mathrm{lo}}\right)$ compared with those in WT tumors, which were mostly naive T cells (CD44-CD62 ${ }^{\text {hi }}$ ) (Figure $1 \mathrm{~K})$. In addition, a larger number of T cells in the Gal1 WT tumors were double-positive for PD1 and Tim-3 and expressed high levels of NFAT-2 (exhausted phenotype, Supplemental Figure 1F) compared with $\mathrm{T}$ cells in the KO tumors. In order to verify that the difference in tumor growth was not due to inherently slower growth kinetics, we performed in vitro cell proliferation assays, which did not show any difference in growth rates between Gal1 WT and Gal1-KO cells over a 72-hour period (Supplemental Figure $1 G)$. To further confirm that the Gal1-mediated effect on tumor growth is mainly driven by suppression of $\mathrm{CD} 8^{+} \mathrm{T}$ cell infiltration, we implanted MOC2 tumors into the CD8A-KO mice (CD8A ${ }^{\text {tm1Mak }}$ mice) (Supplemental Figure $1 \mathrm{H}$ ). In the absence of $\mathrm{CD}^{+} \mathrm{T}$ cells, 
A

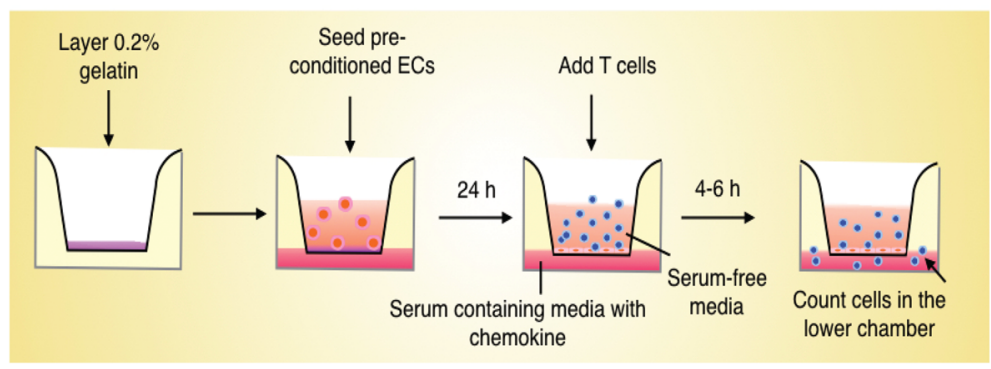

B

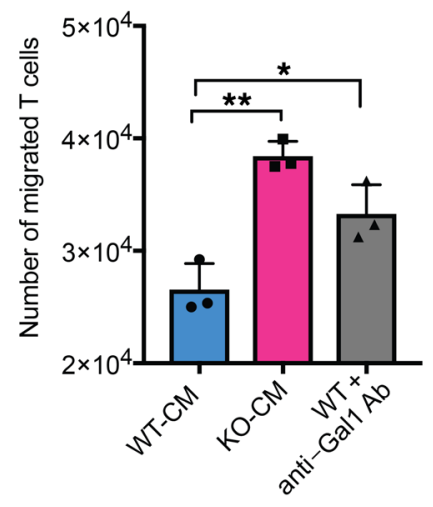

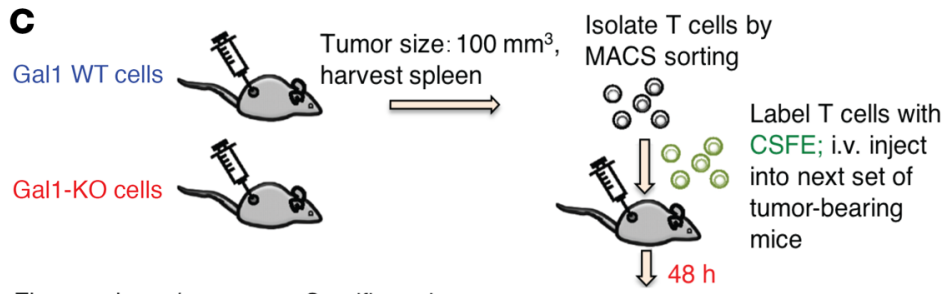

Flow analyses/ $\longleftrightarrow$ Sacrifice mice, 10 min I.V. inject Rhodamine

Cryofreeze section $\longleftarrow$ harvest tumor $\Leftarrow$ dextran and Hoescht 33258 and spleen
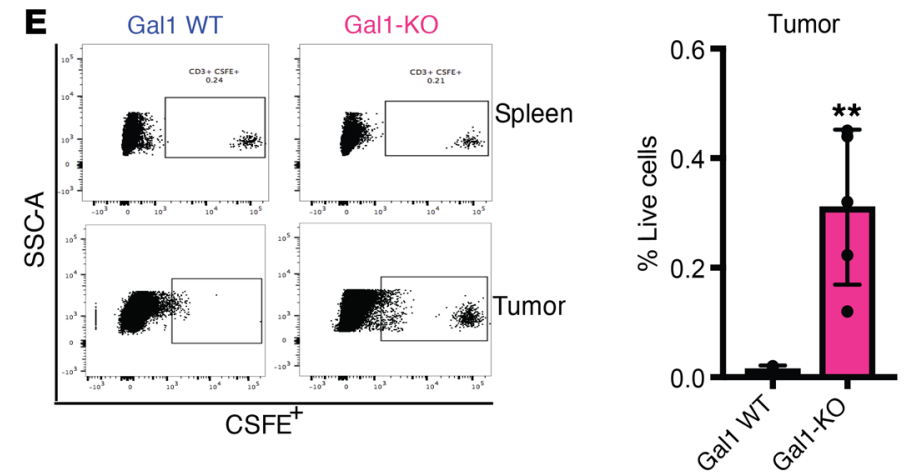

D

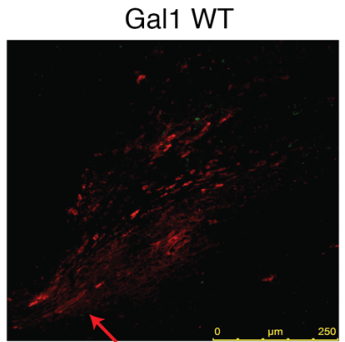

Rhodamine dextran (vessels) / CSFE (T cells)
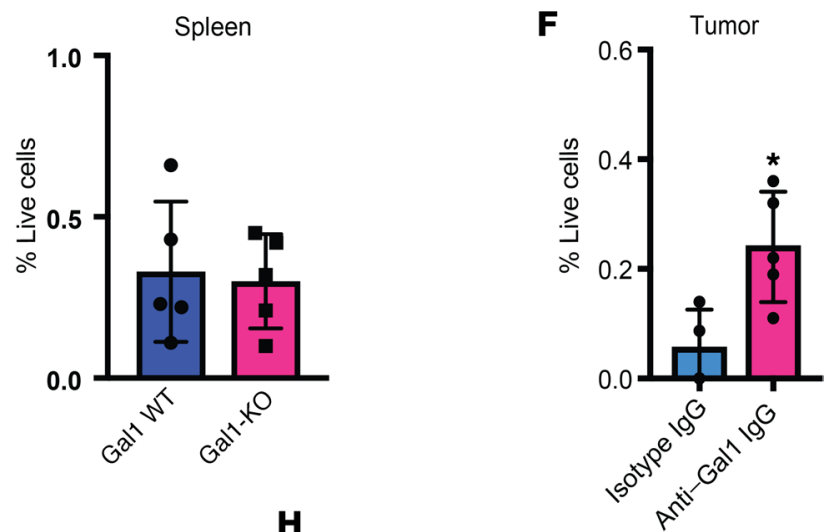

$\begin{aligned} \text { G } \quad \text { WT tumor } & \rightarrow \text { Splenic T cells } \\ \text { KO tumor } & \rightarrow \text { Splenic T cells }\end{aligned}$

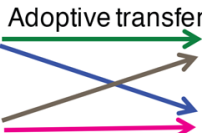

Gal1 WT

tumor-bearing mice

Gal1 KO

tumor-bearing mice

Figure 2. Gal1 mediates $T$ cell exclusion from the tumor microenvironment by inhibiting $T$ cell infiltration. (A) Schematic representation of transendothelial migration assay. (B) Quantification of T cell migration through preconditioned ECs. Mouse ECs (C166) pretreated for 24 hours with MOC2 Gal1 WT or Gal1-KO CM, with or without anti-Gal1 antibody, were seeded onto Transwell inserts. T cells $\left(2 \times 10^{5}\right)$ were seeded onto the upper chamber of the Transwell. The number of migrated cells at the bottom of the well was quantified 4 hours after transfer. (C) Schematic of adoptive T cell transfer experimental design. (D) Representative images of adoptively transferred CSFE-labeled T cells (green) and dextran rhodamine-stained vasculature (red) in MOC2 Gal1 WT or Gal1-KO tumors following cryosectioning and imaging using a $\times 40$ objective (scale bars: $250 \mu \mathrm{m}$ ). (E) Flow cytometric plots and quantification graphs showing the percentage of adoptively transferred live $\mathrm{CD}^{+} \mathrm{T}$ cells in dissociated MOC2 Gal1 WT or Gal1-KO tumors and respective spleens from tumor-bearing mice. SSC-A, side scatter area. (F) Quantification of adoptively transferred T cells in MOC2 Gal1 WT tumors from mice treated for 8 days with isotype IgC or $200 \mu \mathrm{g}$ anti-Gal1 antibody every 4 days. Each dot represents 1 mouse ( $n=4-5$ mice). (G) Schematic representation showing the donor and recipient mice in the adoptive transfer experiments. (H) Quantification of T cells after 48 hours in MOC2 Gal1 WT or Gal1-KO tumors from mice that received splenic T cells from donor mice bearing either MOC1 Gal1 WT or Gal1-KO tumors. ${ }^{*} P<0.05,{ }^{* *} P<0.01$, and ${ }^{* * *} P<0.001$. Each dot represents 2 mice $(n=$ 4-5 mice). Data are presented as the mean \pm SD. A 1-way ANOVA with Tukey's adjustment was used for comparison of multiple treatments (B and $\mathbf{H}$ ); a 2-tailed Student's $t$ test was used for comparison of the single treatment with the control ( $\mathbf{E}$ and $\mathbf{F})$.

H

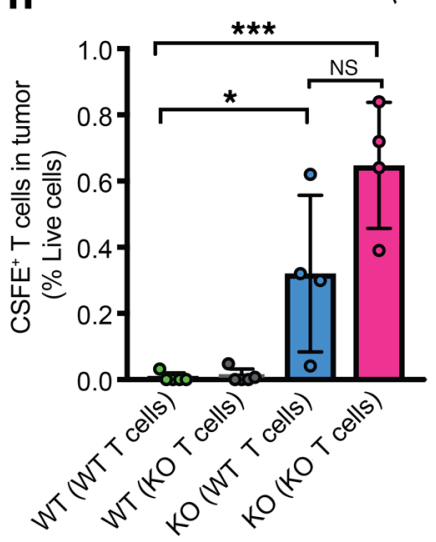


the growth of KO tumors was similar to that of WT tumors except for a modest ( $15 \%)$ growth delay. In tumor immune analyses, Gal1-KO tumors showed increased $\mathrm{CD}^{+} \mathrm{T}$ cells and NK cells in the absence of $\mathrm{CD}^{+} \mathrm{T}$ cells, which may account for the observed modest growth delay (Supplemental Figure 1I). These results confirmed that increased Gal1 expression is associated with poor patient outcomes and that in preclinical models, it promotes tumor growth in a CD8 ${ }^{+} \mathrm{T}$ cell-dependent manner.

Gal1 conditions the tumor endothelium to mediate T cell exclusion. Low $\mathrm{T}$ cell numbers within the tumor can be due to (a) induction of intratumoral $\mathrm{T}$ cell apoptosis, (b) lack of $\mathrm{T}$ cell proliferation within the tumor, and (c) lack of $\mathrm{T}$ cell migration to the tumor. Most of the literature focuses on the direct effect of Gal1 in inducing T cell apoptosis $(25,26)$ using recombinant Gal1 (rGal1). However, the concentration required for Gal1 apoptotic function is quite high, ranging from 7 to $30 \mu \mathrm{M}$, and many reports show inconsistencies in the Gal1 apoptotic effect depending on the tumor model $(21,27,28)$. Like other groups, we found that at least $10 \mu \mathrm{M}$ rGal1 was needed to induce apoptosis in activated murine T cells (Supplemental Figure 2A). Exposure of T cells to MOC2 conditioned medium (CM) $(\times 10$ concentration) led to only $15.75 \%$ apoptosis compared with $8.2 \%$ with concentrated Gal1-KO CM (Supplemental Figure 2B). In addition, TUNEL staining of tumors failed to reveal a significant difference in $\mathrm{T}$ cell apoptosis in the MOC2 model (Supplemental Figure 2C). Therefore, we used more physiologic concentrations of Gal1 to assess its role in creating a barrier to $\mathrm{T}$ cell infiltration by modulating the stromal compartment, specifically the tumor endothelium, which is a known target of Gal1 (29). In a transendothelial migration assay (Figure 2A), we observed a $52 \%$ decrease in the number of activated $\mathrm{T}$ cells that migrated when ECs were preconditioned with concentrated Gal1 WT CM compared with Gal1-KO CM (Figure 2B), and the percentage of migrating $\mathrm{T}$ cells was dependent on the dilution of Gal1 WT CM (Supplemental Figure 2D). The addition of a Gal1-blocking antibody (whose activity with rGal1 was confirmed by both the $\mathrm{T}$ cell apoptosis assay, as shown in Supplemental Figure 2E, and the cell agglutination assay, as shown in Supplemental Figure 2F) to Gal1 WT CM-treated ECs enhanced T cell migration, confirming this function of Gal1 (Figure 2B and Supplemental Figure 2D). To ensure that the CM reflected physiologically relevant secreted Gal1 levels, we measured Gal1 levels in the plasma of patients with HNC $(n=12)$ (Supplemental Figure 2G) and found that these levels were roughly similar to the Gal1 concentration in the concentrated CM (Figure 2B and Supplemental Figure 2D) used in the T cell transendothelial migration study.

To assess the effect of Gal1 on T cell exclusion in vivo, we performed adoptive $\mathrm{T}$ cell transfer experiments, in which $\mathrm{T}$ cells that were harvested from the spleens of mice bearing MOC2 Gal1 WT or Gal1-KO tumors were labeled and infused intravenously into recipient mice bearing the same tumor type and assessed labeled $\mathrm{T}$ cell migration to the tumor and the spleen over a 48-hour period (Figure 2C). Transferred CSFE-labeled T cells were absent in Gal1 WT tumors but were detected in high numbers in Gal1-KO tumors (Figure 2, D and E), whereas the number of labeled splenic $\mathrm{T}$ cells was similar between the 2 groups, suggesting that the effect was tumor specific (Figure 2E, right). We then performed similar adoptive transfer experiments with Gal1 WT tumor-bearing mice, in which they received either isotype IgG or anti-Gal1 antibodies injected intraperitoneally twice weekly before $\mathrm{T}$ cell transfer. We found that Gal1 blockade with an antibody substantially enhanced transferred $\mathrm{T}$ cell infiltration into the tumors (Figure $2 \mathrm{~F}$ ). To address whether the effect of Gal1 was on tumor ECs (TECs) or on T cells, we performed a cross-transfer experiment in which splenic $\mathrm{T}$ cells from Gal1 WT tumor-bearing mice were transferred into Gal1-KO tumor-bearing mice and vice versa (Figure $2 \mathrm{G}$ ). We found that the presence of Gal1 in the tumor microenvironment, rather than on the $\mathrm{T}$ cells, had a greater impact on $\mathrm{T}$ cell tumor infiltration (Figure $2 \mathrm{H}$ ).

Tumor-secreted Gal1 induces chronic STAT activation on endothelium. It is well established that TECs support tumor growth by providing nutrients and oxygen (30). However, recent studies have shown that tumor endothelium can serve as an important barrier to immune cells $(10,18)$. Our in vitro results indicate that exposure to Gal1 could prompt ECs to suppress T cell transmigration. To determine whether the difference in $\mathrm{T}$ cell infiltration between Gal1 WT and Gal1-KO tumors was due to vessel normalization, we assessed pericyte coverage of the tumor vasculature with $\alpha$-smooth muscle actin ( $\alpha$-SMA) staining and vascular leakage with intravenous injection of Hoechst 33258 and rhodamine dextran into tumor-bearing mice. We detected no difference in either pericyte coverage or vessel perfusion between the 2 tumors (Figure 3, A and B). To further verify that this effect was not dependent on vessel perfusion, we isolated TECs from MOC2 Gal1 WT and Gal1-KO tumors, expanded them in culture, and used them for a transendothelial assay. We found that T cell migration across TECs from Gal1 WT tumors was significantly lower than that from Gal1-KO tumors (Figure 3C). Interestingly, PD-L1 levels were substantially higher in TECs from Gal1 WT tumors compared with ECs from either Gal1KO tumors or normal lungs (NECs) (Figure 3D, left). In contrast, cancer cells from Gal1 WT tumors showed lower PD-L1 expression than did cells from Gal1-KO tumors (Figure 3D, right).

To understand how Gal1 affected the global gene expression of ECs, we performed gene expression profiling of TECs isolated from Gal1 WT and Gal1-KO tumors ( $n=3$ /group). Using STRING interaction mapping and pathway analyses (https://string-db. org/), we noted that STAT signaling, especially that of STAT1, was significantly downregulated in Gal1-KO TECs (Supplemental Figure 3, A and B). We also observed a prominent upregulation of the IFN-stimulated gene (ISG) expression signature and immune checkpoint ligands, such as PD-L1 (CD274) and Gal9 (Lgals9), which are STAT1-regulated genes in Gal1 WT TECs (Supplemental Figure 3, A and B).

To determine whether Gal1 directly affected STAT signaling in ECs, we cultured mouse ECs with Gal1 WT or Gal1-KO CM. Gal1-containing CM potently induced the phosphorylation of STAT1 (pSTAT1), which was blunted with anti-Gal1 antibody treatment (Figure 3E). A similar response was noted when HUVECs were treated with human rGal1 at different physiologic concentrations (Figure 3E, right). Since Blouin et al. reported that Gal1 can modulate IFN- $\gamma$ receptor signaling, we decided to examine whether JAK1/2 is involved in this pathway. Indeed, Gal1 increased the phosphorylation of JAK2 in ECs, which is blunted by Gal1 antibody. Moreover, Gal1-mediated STAT1 activation was substantially reduced following treatment with a JAK inhibitor (CAS457081-03-7, Calbiochem) (Figure $3 \mathrm{~F}$ ), suggesting involvement of JAK1/2 in this process. 
A
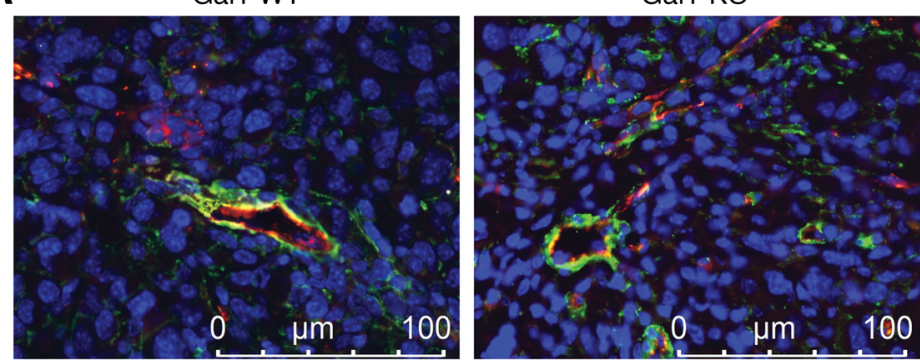

CD31/ $\alpha-S M A$
B

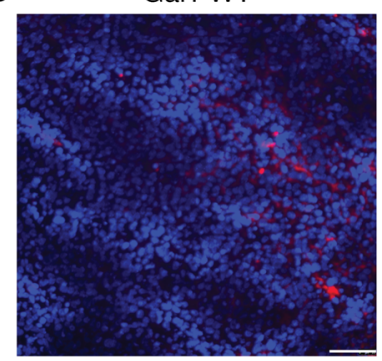

Gal1-KO

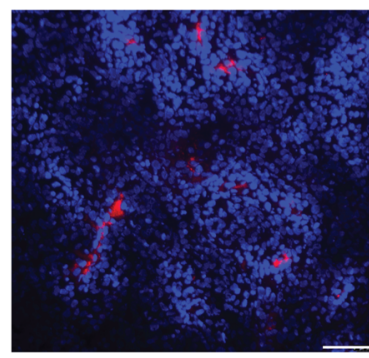

Hoechst 33258 /rhodamine dextran
C

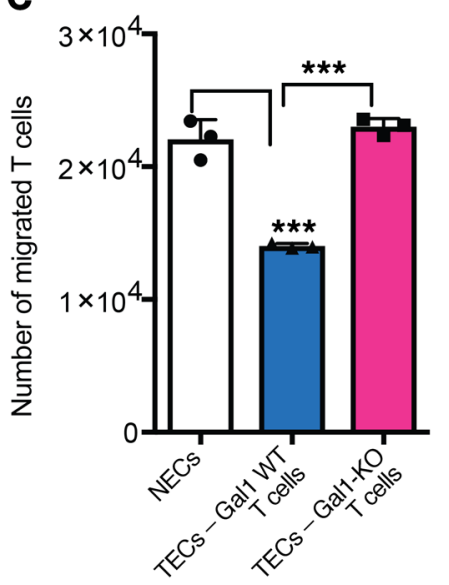

D

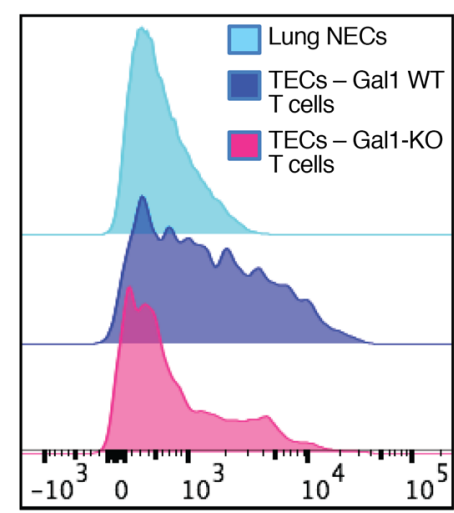

TECS

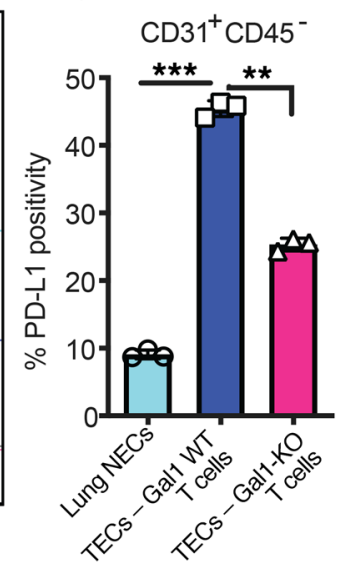

Tumor cells $\mathrm{CD}^{\circ} 1^{-} \mathrm{CD} 45^{-}$
E

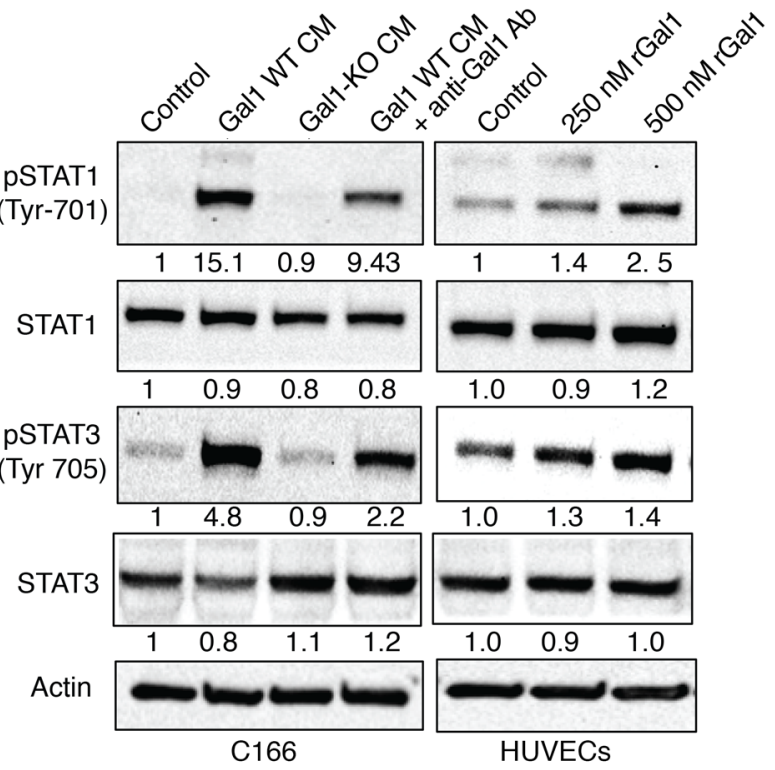

$\mathbf{F}$

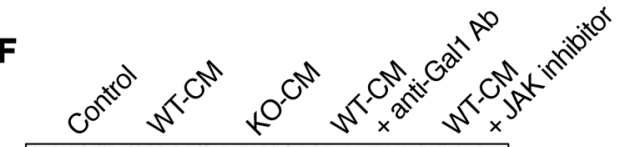

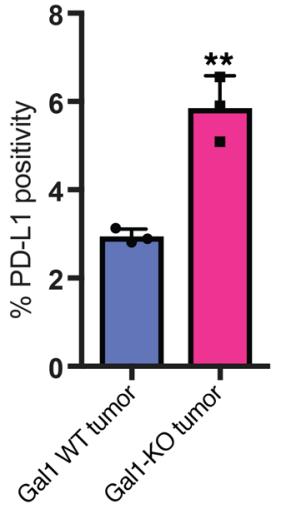
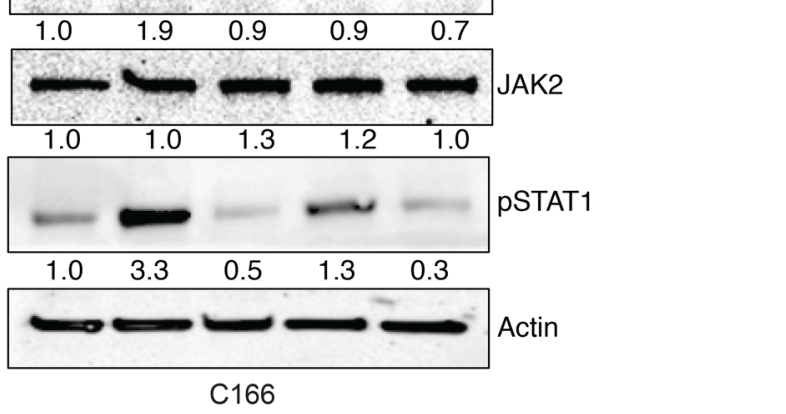

Figure 3. Gal1 preconditioning upregulates STAT1 activation on ECs. (A) Images showing vessel normalization as measured by costaining of CD31 (red) and $\alpha$-SMA (green) in MOC2 Gal1 WT and MOC2 Gal1-KO tumor sections ( $100 \mathrm{~mm}^{3}$ in size). Scale bars: $100 \mu \mathrm{m}$. (B) Images showing vessel perfusion in vivo using intravenous injection of Hoechst 33258 (blue) and rhodamine dextran (red) dyes into Gal1 WT or Gal1-KO tumor-bearing mice at comparable volumes. Data are presented as the mean \pm SD $(n=3)$. Scale bars: $25 \mu \mathrm{m}$. (C) Transendothelial migration of T cells across NECs or TECs isolated from MOC2 Gal1 WT (TECs - Gal1 WT) or Gal1-KO (TECs - Gal1-KO) tumors. (D) Representative histogram and quantification of PD-L1 expression on lung NECs and TECs (CD31+CD45-) and tumor cells (CD31-CD45) isolated from MOC2 Gal1 WT or Gal1-KO tumors. Each dot represents 1 mouse $(n=3)$. (E) Immunoblots of pSTAT1 and total STAT1 in C166 mouse ECs treated with CM from MOC2 Gal1 WT cells, with or without anti-Gal1 antibody, or Gal1-KO cells or HUVECs treated with different concentrations of rGal1 for 3 hours. (F) Immunoblots of pSTAT1, pJAK2, and JAK2 in C166 mouse ECs treated with CM from MOC2 Gal1 WT cells, with or without anti-Gal1 antibody $(10 \mu \mathrm{g} / \mathrm{mL})$ or JAK inhibitor $(100 \mathrm{nM})$, or from Gal1-KO cells treated for 24 hours in $1 \%$ serum-containing media. The numbers below the immunoblots in $\mathbf{E}$ and $\mathbf{F}$ show the relative quantitation of band intensities calculated using $\mathrm{ImageJ}(\mathrm{NIH})$. ${ }^{* *} P<0.01 ;{ }^{* * *} P<0.001$. A 2-tailed Student's $t$ test was used for comparison of the single treatment with the control (D); a 1-way ANOVA with Tukey's adjustment was used for comparison of multiple treatments (C and $\mathbf{D})$. 
A
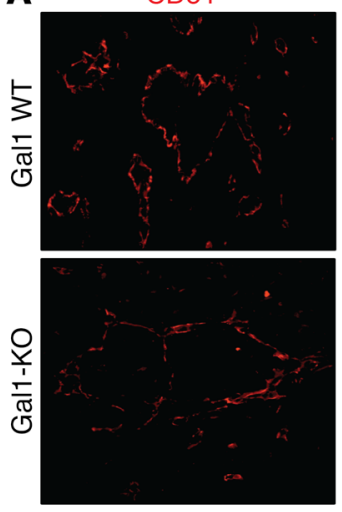

C
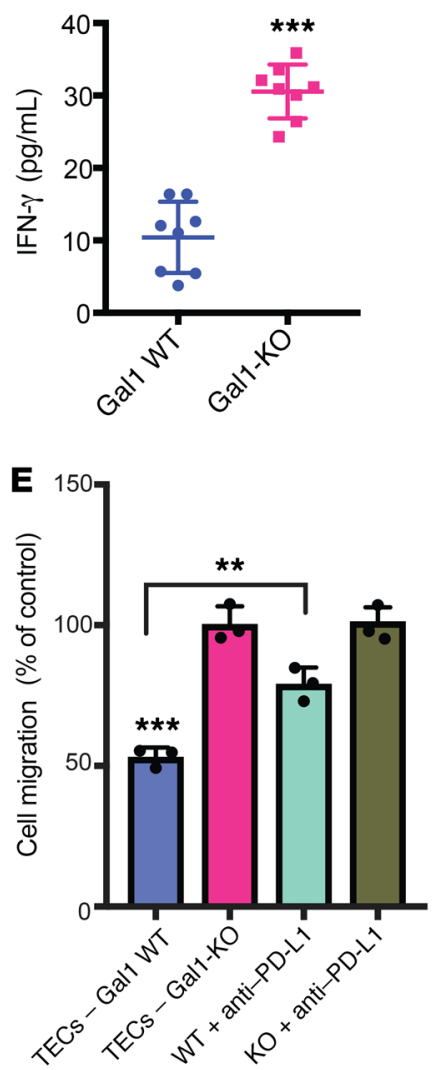

$\mathbf{F}$

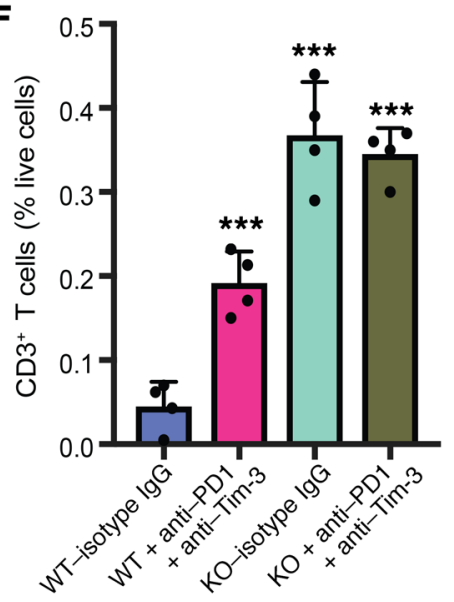

Composite

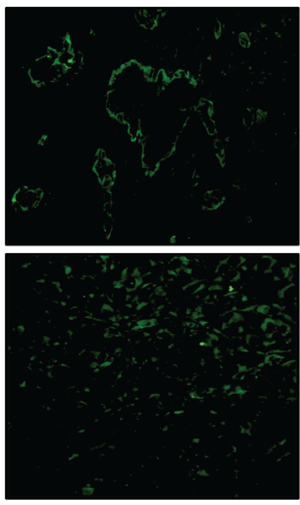

D

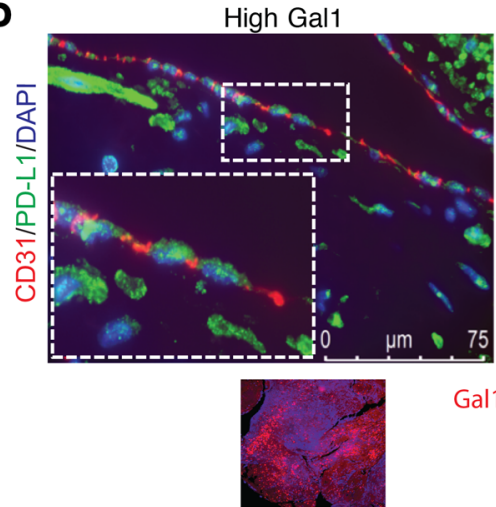

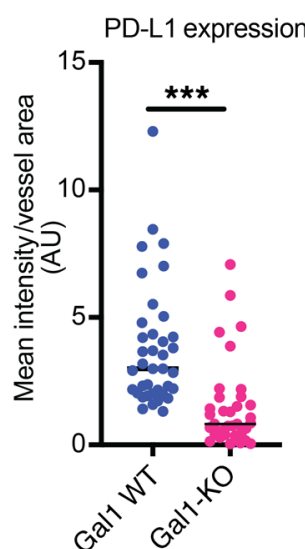

Low Gal1

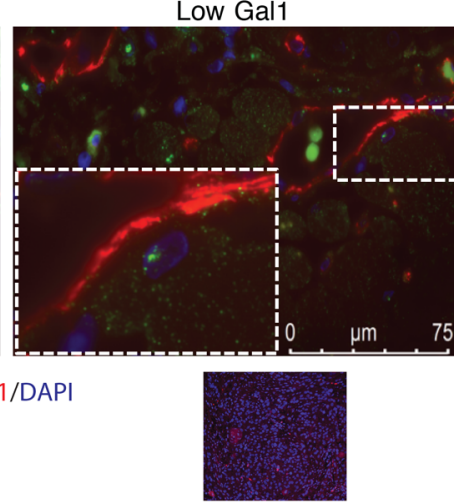

B DAPI/CD31/Gal9
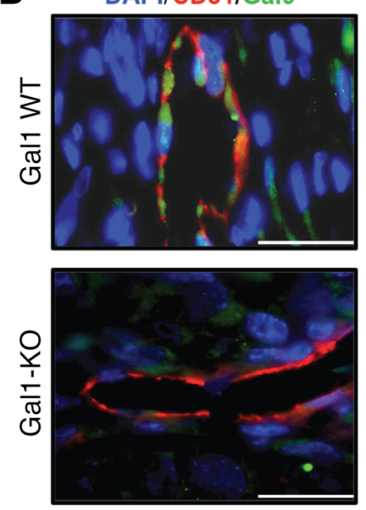

Figure 4. Tumors with high Gal1 levels show expression of enhanced immune checkpoint ligands on ECs. (A) Double-immunofluorescence staining and quantification of PD-L1 on the tumor endothelium in MOC2 Cal1 WT and Cal1-KO tumor sections; CD31 (red) and PD-L1 (green). Scale bar: $20 \mu \mathrm{m}$. (B) Double-immunofluorescence staining for Gal9 in the tumor endothelium of MOC2 Gal1 WT and Gal1-KO tumor sections. CD31 is stained red and Gal9 green ( $n=5$ /group). Scale bar: $20 \mu \mathrm{m}$. (C) Results of ELISA showing the levels of IFN- $\gamma$ in plasma from mice bearing either MOC2 Gal1 WT or Gal1-KO tumors ( $n=8$ /group). (D) Representative images showing differential expression and quantification of PD-L1 on tumor endothelium in human HNSCC with either high or low Gal1 expression ( $n=3$ /group). Original magnification, $\times 10$ (insets). (E) Transendothelial migration of T cells across TECs from MOC2 Gal1 WT or Gal1-KO tumors from mice treated with isotype IgG or anti-PD-L1 antibody. (F) Quantification of cell migration into MOC2 Gal1 WT or Gal1-KO tumors after adoptive transfer of splenic T cells that were blocked with either isotype lgG or anti-PD1 plus anti-Tim-3 antibody for 30 minutes prior to adoptive transfer ( $n=4$ mice/group). Data are representative of experiments repeated at least twice and are presented as the mean $\pm \mathrm{SD}$. ${ }^{*} P<0.01$; ${ }^{* *} P<$ 0.001 . A 2-tailed Student's $t$ test was used for comparison of the single treatment with the control (A, C, and D); a 1-way ANOVA with Tukey's adjustment was used for comparison of multiple treatments (E and $\mathbf{F}$ ).

Tumors secreting high levels of Gal1 show enhanced PD-L1 and Gal9 expression on endothelium, affecting $T$ cell infiltration. We performed immunostaining for PD-L1 and Gal9 in tumor tissues to assess the spatial distribution of these proteins. The expression of PD-L1 and Gal9 in Gal1 WT tumors was primarily restricted to the endothelium, colocalized with CD31, whereas their expression was diffusely distributed throughout the cancer cells in Gal1KO tumors (Figure 4, A and B). This latter finding may suggest that with greater $\mathrm{T}$ cell penetration and increased IFN- $\gamma$ produc- tion (Figure 4C), PD-L1 was induced through an IFN- $\gamma$-adaptive immune resistance mechanism in KO tumors. We observed similar PD-L1 staining patterns for human HNC samples with high and low Gal1 expression (Figure 4D). Coculturing murine ECs with MOC2 Gal1 WT cells revealed substantial increase in PD-L1 and Gal9 protein expression, which we did not observe in ECs cocultured with Gal1-KO tumor cells (Supplemental Figure 4A). To confirm that PD-L1 expression on tumor endothelium contributes to the effect of Gal1 on transendothelial migration of T cells, 
A

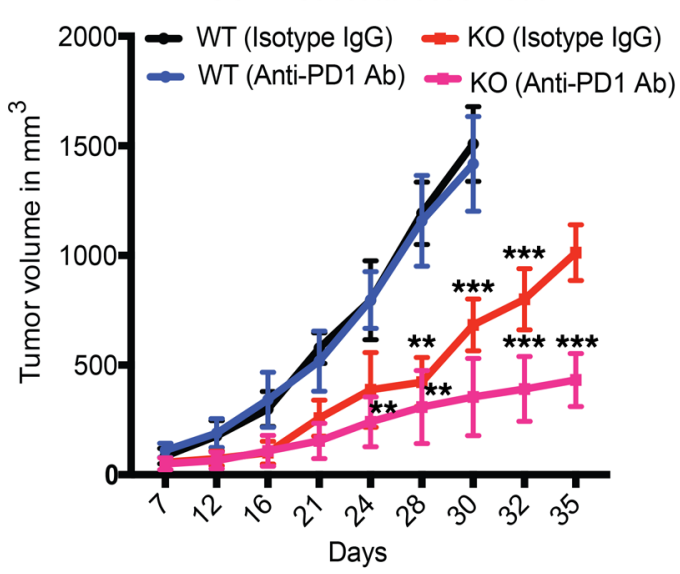

C

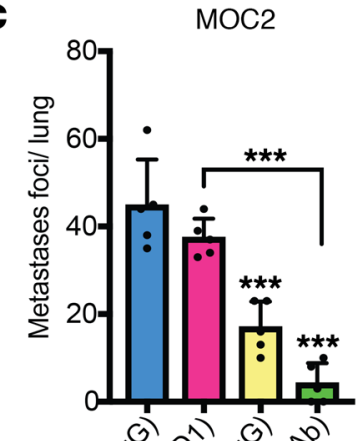

1000 की को

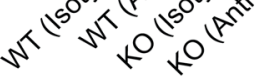

D

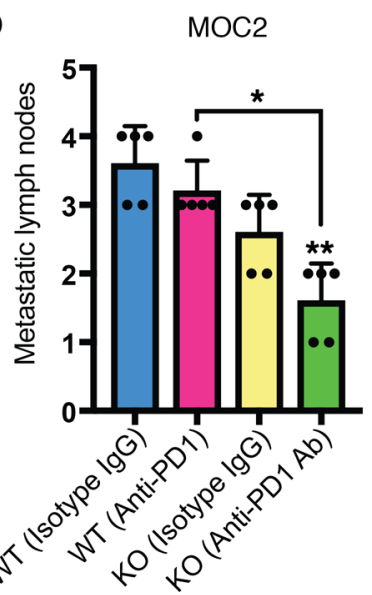

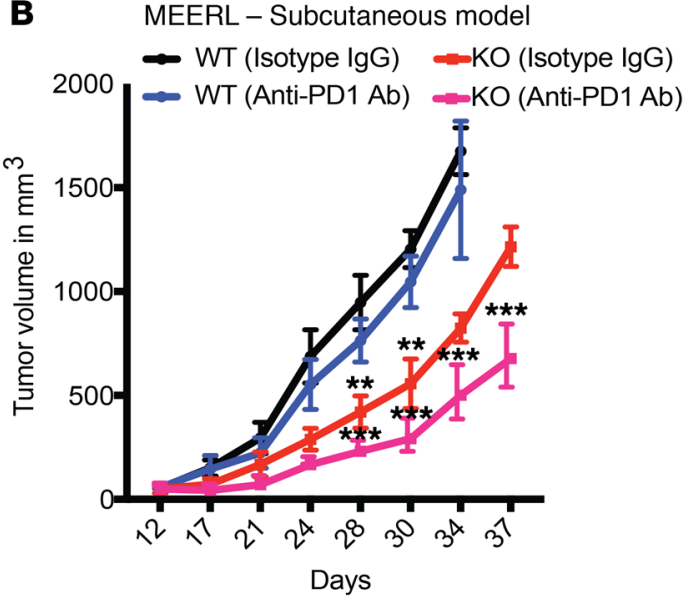

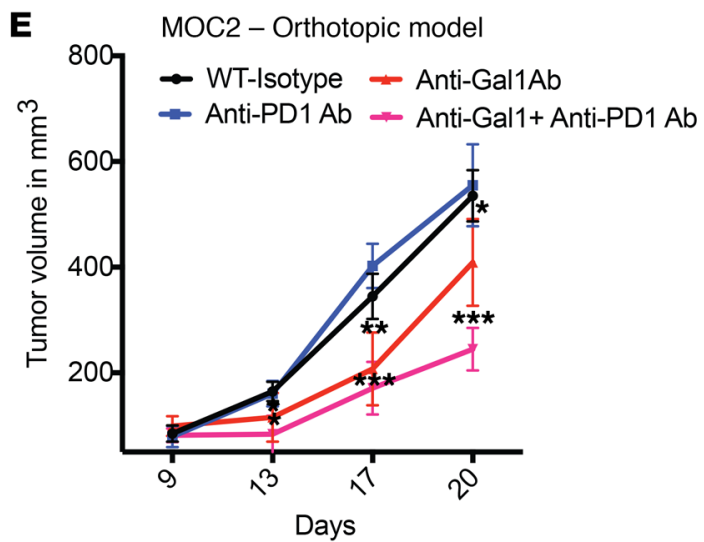

$\mathbf{F}$

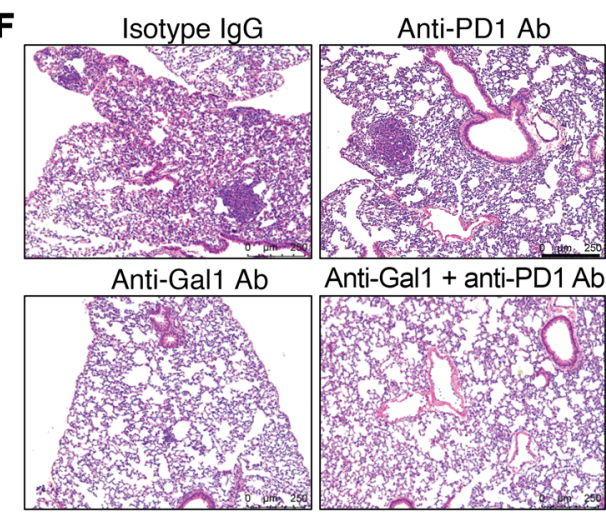

H

Gal1 staining on HNSCC patient biopsies High Gal1

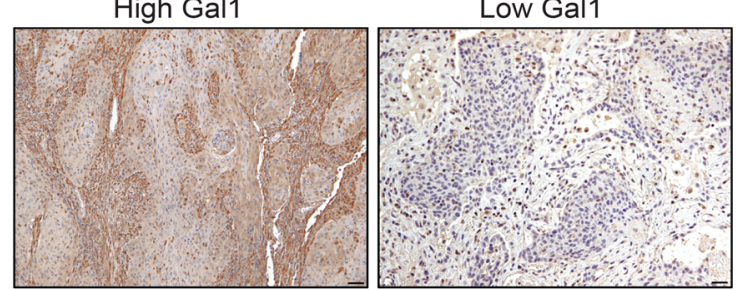

Gal1/Hematoxylin
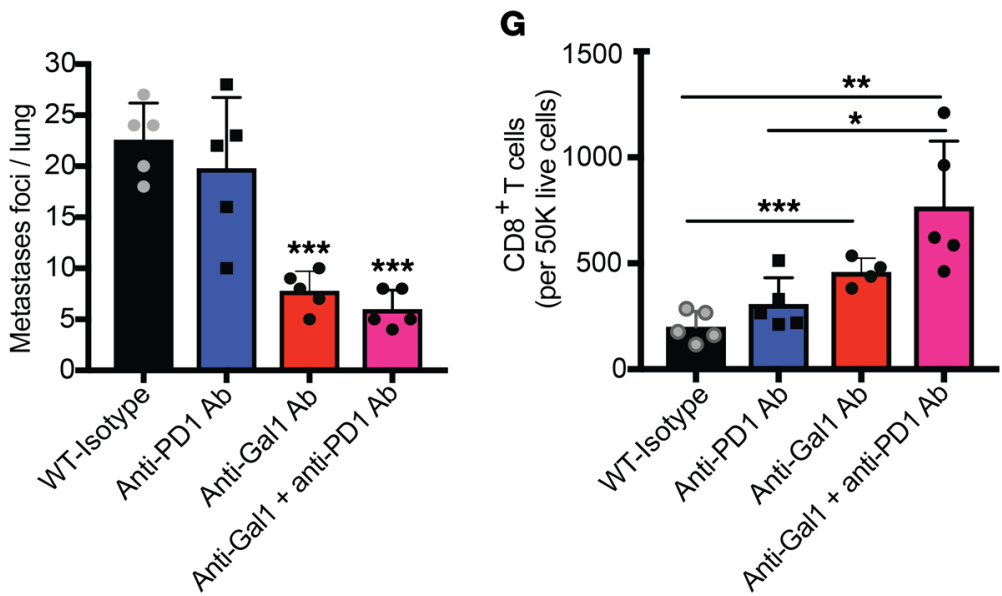

I Patient survival analyses after anti-PD1 therapy

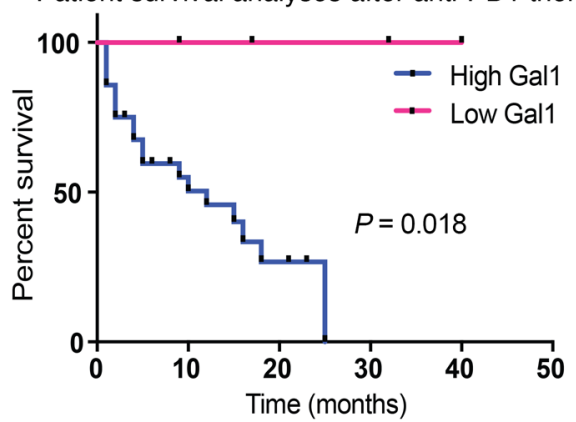


Figure 5. Gal1 inhibition reverses PD1 blockade resistance in a HNC model. Tumor growth curves in C57BL/6 mice subcutaneously implanted with (A) MOC2 Gal1 WT or Gal1-KO tumor cells $\left(2.5 \times 10^{5}\right)$ or (B) MEERL Gal1 WT or Gal1-KO tumor cells $\left(1 \times 10^{6}\right)$. Following tumor establishment $(\sim 75$ $\mathrm{mm}^{3}$ ), mice were treated with either isotype IgC or anti-PD1 antibody (200 $\mu$ i.p. every 4 days) for 4 weeks. (C) Quantification of lung metastatic foci at the end of treatment in mice bearing MOC2 tumors. (D) Number of inguinal (left and right) and axillary (left and right) nodal metastases in each mouse for each treatment group. Each dot represents 1 mouse ( $n$

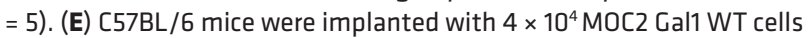
in the buccal cavity (orthotopic), followed by treatment with isotype IgC or anti-Gal1 antibody ( $150 \mu$ g, i.p.) and/or anti-PD1 antibody ( $200 \mu$ g, i.p.) every 4 days. Tumor growth was measured at regular intervals using a caliper ( $n=5-8$ mice/group). (F) Representative images and quantification of lung metastatic foci after treatment in the MOC2 orthotopic model ( $n$ $=5$ mice/group). Scale bars: $250 \mu \mathrm{m}$. (G) Quantification of CD8 ${ }^{+} \mathrm{T}$ cells in orthotopically implanted tumors after treatment. (H) Representative images showing immunohistochemical staining for Gal1 in biopsy samples from patients with HNSCC prior to immunotherapy treatment. Stainings were used for the grading of high or low Gal1 expression levels. Scale bar: $100 \mu \mathrm{m}$. (I) Kaplan-Meier analysis showing survival probability based on the expression of Gal1 protein in the tumor cells and tumor stroma of patients ( $n=33$ patients) with recurrent/metastatic HNC treated with immune checkpoint therapy. High Gal1 (high Gal1 levels in either the tumor or stroma); low Gal1 (low Gal1 levels in both the tumor and stroma). Data are presented as the mean $\pm \mathrm{SD} .{ }^{*} P<0.05,{ }^{* *} P<0.01$, and ${ }^{* * *} P<0.001$. A 1-way ANOVA with Tukey's adjustment was used for comparison of multiple treatments (C, $\mathbf{D}, \mathbf{F}$, and $\mathbf{G}$ ); a repeated-measures ANOVA was used for measurement of tumor growth over time (A, B, and E). Overall survival was summarized using Kaplan-Meier curves, and the groups were compared using log-rank tests (I). The rates of response to immunotherapy and distribution of Gal1 staining were analyzed using a $\chi^{2}$ test (I).

we performed a transmigration assay, in which we layered the Transwell with Gal1 WT or Gal1-KO TECs and incubated these cells with PD-L1-blocking antibody for 1 hour. The blocking antibody was then washed off, followed by the addition of activated splenic T cells from MOC2 tumor-bearing mice (Supplemental Figure $4 \mathrm{~B}$ ). We found that PD-L1 blockade on ECs significantly rescued $(30 \%)$ the migration of $\mathrm{T}$ cells across the endothelium (Figure 4E). For in vivo validation of these findings, we preincubated $\mathrm{T}$ cells isolated from tumor-bearing mice with the blocking antibodies anti-PD1 and anti-Tim-3 (receptors for PD-L1 and Gal9, respectively) prior to adoptive transfer into Gal1 WT and Gal1-KO tumor-bearing mice. Blocking of PD1 and Tim-3 on T cells partially improved T cell infiltration into Gal1 WT tumorbearing mice (Figure $4 \mathrm{~F}$ ), confirming that the induction by Gal1 of immune-inhibitory ligands (PD-L1 and Gal9) on ECs can affect $\mathrm{T}$ cell immigration into the tumor.

Gal1 inhibition reverses PD1 blockade resistance in HNC. Since MOC2 and MEERL tumors were deficient in T cells, we hypothesized that these tumors would not respond to anti-PD1 therapy and that Gal1 deletion or blockade with an antibody would enhance the effectiveness of anti-PD1 therapy in these tumors by improving $\mathrm{T}$ cell infiltration. We found that anti-PD1 antibody alone had no effect on MOC2 tumor growth when implanted subcutaneously (Figure 5A). However, knocking out Gal1 led to a substantial improvement in tumor growth delay in response to antiPD1 therapy. Similarly, anti-PD1 treatment had a minimal effect on $\mathrm{HPV}^{+}$MEERL tumors, but knocking out Gal1 with PD1 blockade led to a significant reduction in tumor growth (Figure 5B).
We quantified lung and nodal metastases in the MOC2 model when the vector control SQ tumors reached $1500 \mathrm{~mm}^{3}$ in size. Gal1 KO led to a significant reduction in spontaneous lung metastases that was further improved with anti-PD1 antibody treatment, resulting in an approximately $88 \%$ reduction in metastatic burden compared with anti-PD1 treatment in Gal1 WT tumor-bearing mice (Figure 5C). Likewise, Gal1 deletion combined with antiPD1 therapy led to fewer nodal metastases in the draining nodal compartment (bilateral inguinal and axillary lymph nodes [LNs]) compared with PD1 treatment alone (Figure 5D). We also implanted MOC2 tumors orthotopically into the buccal cavity and treated the mice with anti-PD1 and/or anti-Gal1 antibody (tumors size of approximately $50-75 \mathrm{~mm}^{3}$, administered i.p. at $200 \mu \mathrm{g}$ every 4 days until sacrifice, on approximately day 20). Combined Gal1 and PD1 blockade with antibodies resulted in a 54\% reduction in tumor growth at sacrifice compared with the isotype control- or anti-PD1-treated group (Figure 5E). Similarly, we observed a 74\% reduction in spontaneous lung metastatic burden in the combine antibody-treated group compared with the isotype control group in this orthotopic model (Figure 5F). Flow cytometric analysis of dissociated orthotopic tumors showed that the combined treatment boosted $\mathrm{CD} 8^{+} \mathrm{T}$ cell numbers (Figure $5 \mathrm{G}$ ).

To determine the clinical relevance of these findings, tumor tissues from 33 patients who had received anti-PD1 therapy to treat recurrent/metastatic HNCs were stained for Gal1 protein expression using immunohistochemistry. Patient characteristics and treatment details are shown in Table 1. Among these patients, 25 had squamous cell carcinoma histology, 4 of which were $\mathrm{HPV}^{+}$. On the basis of the staining pattern (Figure $5 \mathrm{H}$ ), we grouped patients into 3 different risk groups: high risk (high Gal1 expression in both tumor cells and surrounding stroma), intermediate risk (high expression in either tumor cells or stroma), and low risk and (low expression in both). The rate of response (based on the response evaluation criteria in solid tumors [RECIST]) to anti-PD1 treatment was $67 \%$ in the low-risk group, $9 \%$ in the intermediate-risk group, and $0 \%$ in the high-risk group $(P=0.053)$. Excluding the 2 patients who terminated anti-PD1 therapy prematurely due to toxicity and using the number of PD1 treatment cycles as a surrogate for treatment benefit, the median number of treatment cycles was 12 for the low-risk group, 3 for the intermediate-risk group, and 4 for the high-risk group $(P$ $=0.0053)$. Since there was no difference in survival after initiation of anti-PD1 therapy between the intermediate- and high-risk groups (Supplemental Figure 5), we combined their results. Overall survival after anti-PD1 treatment was significantly better for the low-risk group compared with survival for the combined intermediate- and high-risk groups $(P=0.018$, Figure $5 \mathrm{I})$. Of note, all $4 \mathrm{HPV}^{+}$tumors were in the Gal1 high-risk group, 18 of $21 \mathrm{HPV}^{-}$tumors were in the Gal1 high-risk group, and 3 of 21 tumors were in the Gal1 low-risk group, respectively. We also stained for $\mathrm{CD} 8^{+} \mathrm{T}$ cells in these tumors to establish the relationship between Gal1 levels, $\mathrm{CD}^{+} \mathrm{T}$ cell infiltration, and immunotherapeutic response. We primarily scored Gal1 stromal expression in the center of the tumor and found that, among tumors with high Gal1 stromal staining, 50\% (13 of 26) had very low $\mathrm{CD} 8$ staining. In contrast, among tumors with low Gal1 stromal staining, $71 \%$ (5 of 7 ) showed high CD8 staining (Table 2). We were not able to perform multivariate analysis due to the small number of patients in the cohort, especially in the low-risk group. 


\section{Table 1. Patient characteristics}

\begin{tabular}{|c|c|}
\hline Patient characteristics & Number $(n)$ \\
\hline Total & 33 \\
\hline Sex & 18 females/15 males \\
\hline Age: median (range) & $65(34-85)$ \\
\hline \multicolumn{2}{|l|}{ Histology } \\
\hline Squamous cell carcinoma & $25(76 \%)$ \\
\hline Nasopharynx carcinoma & $1(3 \%)$ \\
\hline Neuroendocrine carcinoma & $2(6 \%)$ \\
\hline Adenoid cystic carcinoma & $2(6 \%)$ \\
\hline Acinic cell carcinoma & $1(3 \%)$ \\
\hline Papillary thyroid carcinoma & $1(3 \%)$ \\
\hline Mesothelial melanoma & $1(3 \%)$ \\
\hline \multicolumn{2}{|l|}{ Gal1 risk group } \\
\hline Low & $5(15 \%)$ \\
\hline High & $28(85 \%)$ \\
\hline Squamous cell carcinoma & $25 / 33$ \\
\hline $\mathrm{HPV}^{+}$ & $4 / 33(24 \%)$ \\
\hline Gal1 (low risk) & $0(0 \%)$ \\
\hline Gal1 (high risk) & $4(100 \%)$ \\
\hline HPV $^{-}$ & $21 / 33(72 \%)$ \\
\hline Gal1 (low risk) & $3(14.2 \%)$ \\
\hline Gal1 (high risk) & $18(85.7 \%)$ \\
\hline \multicolumn{2}{|l|}{ Anti-PD1 therapy } \\
\hline Nivolumab & $10(30 \%)$ \\
\hline Pembrolizumab & $23(70 \%)$ \\
\hline No. of anti-PD1 Ab infusion cycle: median (range) & $3(1-19)^{A}$ \\
\hline \multicolumn{2}{|l|}{ Response } \\
\hline Yes & $4(12 \%)$ \\
\hline No & $27(82 \%)$ \\
\hline Unknown & $2(6 \%)$ \\
\hline \multicolumn{2}{|l|}{ Survival status at last follow-up } \\
\hline Alive & $9(27 \%)$ \\
\hline Dead & $20(61 \%)$ \\
\hline Unknown & $4(12 \%)$ \\
\hline Follow-up duration: median (range) & 7.6 months (0.13-40.9) \\
\hline
\end{tabular}

Combining Gal1 blockade with radiotherapy significantly improves the response to immunotherapy. We previously showed that radiotherapy (RT) increased Gal1 secretion from different cancer cell types, including HNC cells (31). In addition, anti-PD1 therapy has been shown to enhance the effectiveness of RT in HNC, and the combination of RT and anti-PD1 is currently being tested in clinical trials for locally advanced HNC. Therefore, we were interested to see whether Gal1 blockade could further improve the results of RT and anti-PD1 therapy. Once subcutaneous MOC2 Gal1 WT and Gal1-KO tumors reached $100 \mathrm{~mm}^{3}$ in size, we treated them with 6 fractionated 2.5-Gy doses, with administration of anti-PD1 antibodies beginning 1 day prior to RT (32). Antibodies were injected intraperitoneally every 4 days for 30 days. Anti-PD1 alone with RT showed minimal improvement in Gal1 WT tumors; however, in the Gal1-KO tumors, the combination showed a greater antitumor response, with $70 \%$ of the mice having no detectable tumor 50 days after RT initiation
(Figure 6A). Consistent with our hypothesis, evaluation of the tumor immune infiltrates revealed that the effect was primarily due to an increase in T cells and DCs with the combined treatment (Figure 6, B and C). We also tested therapeutic blockade using the anti-Gal1 antibody along with RT and an anti-PD1 antibody in an orthotopic tumor model. We recapitulated the effects of tumor growth inhibition, with the combination antibody treatment being the most effective (Figure 6D).

\section{Discussion}

Recent studies have suggested that angiogenesis and immune suppression go hand in hand and that tumor vasculature plays an important role in channeling immune cells to the tumor, with channeling being an important step $(33,34)$. Our data reveal a significant function of Gal1 that has not to our knowledge been previously explored. When tumors are small, low levels of secreted Gal1 can activate STAT1 in TECs, leading to high expression of PD-L1 and Gal9 proteins on the endothelial barrier. The findings indicate that this high expression acts as a mechanism of T cell exclusion by inhibiting transmigration vis-à-vis tumor cell paracrine signaling, thereby promoting tumor immune escape. Previously, the tumor endothelium was viewed as a passive physical barrier to immune cell infiltration. In agreement with some recent studies $(10,18)$, we have shown that tumor endothelium could function as an active partner in establishing immune privilege through upregulation of immune-inhibitory ligands. Our study, along with others, reveals that TECs are malleable to microenvironmental cues, including tumor-secreted factors, that can condition them into potent mediators of immunosuppression (35).

Second, we believe our findings can explain the controversy in the literature regarding the proapoptotic effect of Gal1 on $\mathrm{T}$ cells $(17,21)$, especially in the context of tumor physiology. The debate stems primarily from the very high Gal1 concentrations required to trigger $\mathrm{T}$ cell apoptosis and the variable results in tumor models and cell types $(20,27)$. Gal1 mediation of T cell apoptosis may occur in tumors once they reach a large enough size to produce the high levels of Gal1 that are required for this function. However, our data suggest that early in tumor development, Gal1 mediates T cell exclusion through its effect on the endothelium-immune interface.

Although we showed that Gal1 activated the JAK/STAT signaling pathway in these cells, the mechanism of this activity needs further exploration. One possible explanation is that Gal1 binding to glycosylated sites on IFN- $\gamma$ receptor (IFN- $\gamma$ R) leads to activation of the JAK/STAT signaling pathway. Blouin and colleagues have shown that Gal1 can bind directly to IFN- $\gamma$ R1 and -2 , which could lead to modulation of JAK/STAT pathway function (36). Interestingly, it was recently shown that chronic IFN- $\gamma$ signaling via STAT

\section{Table 2. Distribution of CD8+ $\mathbf{T}$ cells in pretreatment tumor samples by Gal1 stromal staining in the center of the tumor}

$\begin{array}{lccc}\text { Gal1 staining in stroma } & \text { Low CD8+ } & \text { staining }(\boldsymbol{n}) & \text { High CD8+ } \\ \text { High } & 13 & 13 & 26 \\ \text { Low } & 2 & 5 & 7\end{array}$


A

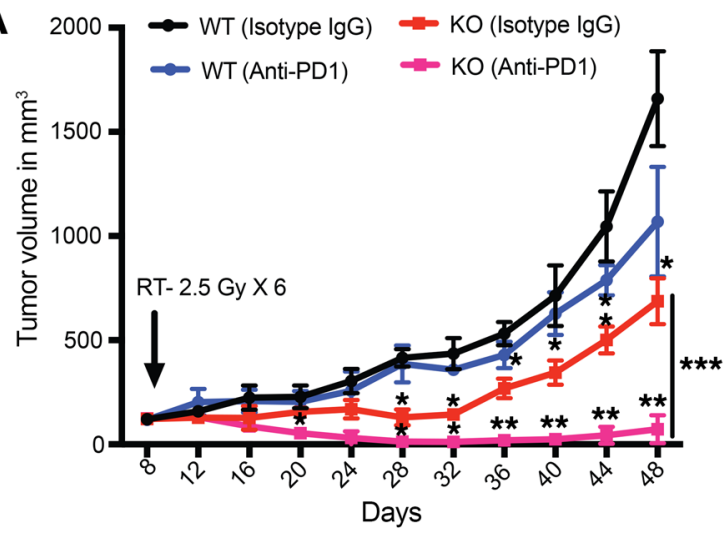

D

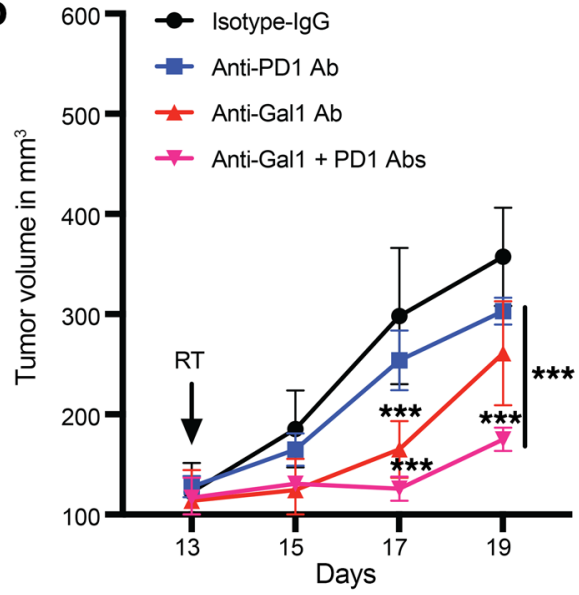

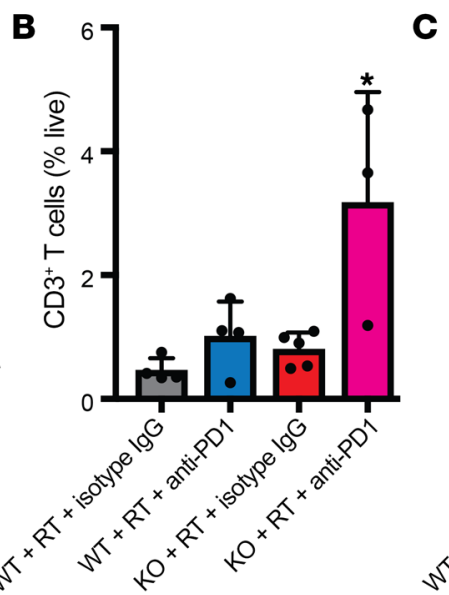

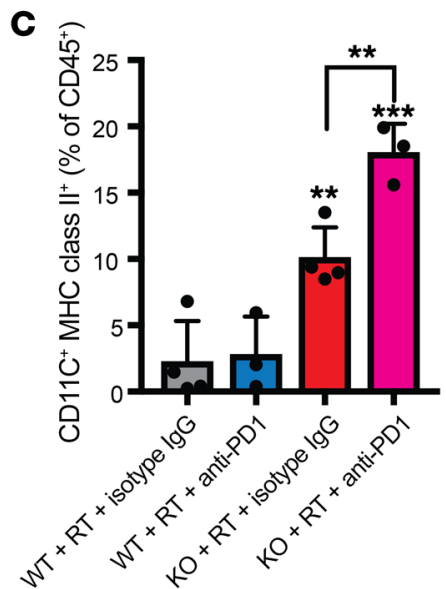

Figure 6. Combining Gal1 blockade with RT significantly improves the response to immunotherapy. (A) Tumor growth curve for MOC2 Gal1 WT or Gal1-KO tumors treated with $15 \mathrm{~Gy}(2.5 \mathrm{~Gy} \times 6)$ radiation and anti-PD1 antibody ( $n=5$ mice/group). (B) Flow cytometric analyses of dissociated tumors showing the percentage of $\mathrm{CD}^{+} \mathrm{T}$ cells after treatment. (C) Percentage of CD11 $\mathrm{C}^{+} \mathrm{MHC}$ class $\mathrm{II}^{+} \mathrm{DCs}$. (D) Tumor growth curve for MOC2 Gal1 WT tumors treated with $15 \mathrm{~Gy}(2.5 \mathrm{~Gy} \times 6$ ) focused radiation with or without anti-Gal1 or anti-PD1 antibody $(n=4-5)$. Data are presented as the mean \pm SD. ${ }^{*} P<0.05$, ${ }^{*} P<$ 0.01 , and ${ }^{* *} P<0.001$. A repeated-measures ANOVA was used for measurement over time of tumor growth (A and D); a 1-way ANOVA with Tukey's adjustment was used for comparing multiple treatments (B and $\mathbf{C}$ ).

signaling could lead to consistent upregulation of ISG footprints, which was in turn associated with resistance to immunotherapy (37). Our study points toward a similar phenotype, in which induction of ISGs via chronic STAT signaling on tumor endothelium may similarly affect tumor immune responses. Another possible mechanism is the binding of Gal1 to the N-glycan of VEGFR2, activating VEGF signaling (38). However, we did not observe a significant difference in vessel normalization or perfusion at the early stages of growth of MOC2 Gal1 WT or Gal1-KO tumors.

In this study, we identify an important mechanism of Gal1mediated immune evasion through the endothelial compartment that can significantly affect antitumor immune responses. These data suggest that Gal1 can mediate resistance to both antiangiogenic agents and ICIs, which was recently confirmed in a clinical report showing that increases in circulating Gal1 were associated with poor clinical outcomes during combined anti-VEGF and anti-CTLA-4 therapy, whereas elevated antibody responses to Gal1 were associated with favorable clinical outcomes with these treatments (39). Our data support these findings, as Gal1 blockade enhanced the effect of anti-PD1 therapy in 2 preclinical models. In addition, lack of Gal1 in the tumor and stroma was linked to better responses to anti-PD1 therapy and higher survival rates in a small cohort of patients with HNC treated with this therapy for recurrent/metastatic disease. These data will need validation in a larger, independent cohort of prospectively treated patients; but if validated, these findings would suggest that Gal1 expression may be used to select patients for ICI therapy and that Gal1 targeting can improve the efficacy of these treatments.
Anti-PD1 therapy is being tested in combination with RT with or without chemotherapy in patients with locally advanced HNC in several large phase III randomized trials (e.g., NCT02764593, ClinicalTrials.gov). Since radiation can increase the secretion of Gal1 (31), we hypothesized that Gal1 blockade could enhance the efficacy of radiation plus anti-PD1 therapy, and our hypothesis was confirmed in the MOC2 model. Gal1 blockade using a therapeutic antibody did not result in any outward signs of toxicity in the animals tested, which further emphasizes the idea that Gal1 could be a safe target. In summary, our results indicate that Gal1 can contribute to tumor immune privilege by mediating $\mathrm{T}$ cell exclusion from the tumor. The study further emphasizes Gal1 as a target that bridges immune suppression and tumor angiogenesis, shedding light on a unique approach to improve the results of immunotherapy in HNC.

\section{Methods}

Cell lines and culture conditions. HPV murine oral cancer cells (MOC1 and MOC2) were provided in-house and maintained in culture in IMDM/F12 (2:1) with 5\% FCS, 1\% penicillin-streptomycin, $2 \mu \mathrm{g} / \mathrm{mL}$ amphotericin, $5 \mathrm{ng} / \mathrm{mL}$ EGF (MilliporeSigma), $400 \mathrm{ng} /$ $\mathrm{mL}$ hydrocortisone, and $5 \mu \mathrm{g} / \mathrm{mL}$ insulin. The $\mathrm{HPV}^{+}$murine oral cancer cells (MEERL) were provided by William Spanos (Sanford Research, Sioux Falls, South Dakota, USA). The cells were grown in DMEM/Ham's F-12 (2:1), containing 10\% FBS, $25 \mu \mathrm{g} / \mathrm{mL}$ hydrocortisone, $5 \mu \mathrm{g} / \mathrm{mL}$ transferrin, $5 \mu \mathrm{g} / \mathrm{mL}$ insulin, $1.36 \mathrm{ng} / \mathrm{mL}$ triiodo-thyronine, and $5 \mathrm{ng} / \mathrm{mL}$ EGF. For CM collection experiments, serum-free media containing $55 \mu \mathrm{M} \beta$-mercaptoethanol was used. 
HUVECs were obtained from the American Type Culture Collection (ATCC) and expanded with the EGM-2MV Medium Bullet Kit (Lonza), using HUVECs from passages 3 to 7. The murine EC lines C166 and SVEC were obtained from the ATCC and grown in complete EC medium (catalog M1166, Cell Biologics). Stable Gal1KO cells were generated with CRISPR/Cas9 targeting using pSpCas9(BB)-2A-Puro (PX459) V2.0 (plasmid 62988) from Addgene and sgRNA designed to target murine Gal1. Gene disruption was validated by Western blotting and DNA-Seq. For overexpression of Gal1 in MOC1 cells, lentiviral overexpression vectors were custom synthesized by VectorBuilder. The primer sequences used in the study are listed in Supplemental Table 1.

In vivo experiments. Seven- to ten-week-old C57BL/6 mice were purchased from Charles River Laboratories. To generate subcutaneous tumors, $2.5 \times 10^{5}$ (MOC2) or $1 \times 10^{6}$ (MOC1 or MEERL) cells were injected into the right flank region of the mice. For the orthotopic model of MOC2; $4 \times 10^{4}$ cells were injected into the buccal cavity of the mice. Tumor growth was measured every 3 days using a vernier caliper until euthanization. Mice were euthanized, and tumors were surgically removed along with spleen, draining LNs, and lungs. Tumor tissues used for Western blotting were snap-frozen. Tissues used for immunohistochemical analysis were fixed with $10 \%$ neutral buffered formalin (NBF) overnight and then stored in $70 \%$ ethanol until paraffin fixation. Tissues for flow cytometric analysis were collected in RPMI media and processed immediately. LNs and lungs were analyzed for metastases using H\&E staining.

Gal1 ELISA. MOC1, MEERL, and MOC2 cell lines were cultured to $70 \%$ confluency in regular growth media. The cells were changed to serum-free media and subjected either to normoxic or hypoxic $(0.5 \%$ $\mathrm{O}_{2}$ ) conditions for 24 hours. After 24 hours, the $\mathrm{CM}$ was collected and concentrated $\times 10$ using a Amicon Ultra $10 \mathrm{~K}$ centrifugal filter device. The CM was diluted by normalization to cell numbers in each sample and then subjected to a mouse Gal1 ELISA according to the manufacturer's instructions (DY1245, R\&D Systems). For Gal1 measurements in mouse plasma, whole blood samples from retro-orbital bleeds were collected in BD Microtainer tubes with EDTA and centrifuged at 1200 $g$ for 10 minutes. Plasma was diluted and the Gal1 concentration estimated using the standard protocol. For human samples, patient blood collected in heparinized tubes was centrifuged at $3000 \mathrm{rpm}$ for 10 minutes. Gal1 levels were measured using a human Gal1 ELISA kit (DGAL10, R\&D Systems).

Antibodies for in vivo experiments. Antibodies against Gal1 and PD1 were a gift from Bristol-Myers Squibb. Rat IgG2a, mouse IgG2a isotype antibodies, and anti-Tim-3 (BE0115, clone RMT3-23) antibodies were obtained from BioXcell.

Radiation treatment. Mice were randomized to different treatment groups on the basis tumor size to ensure that all treatment groups started with similar tumor sizes. Tumors between the sizes of 75 and 100 $\mathrm{mm}^{3}$ were irradiated. Volume measurements were obtained every 2 to 3 days after initiation of treatment using $\mathrm{V}=\mathrm{L} \times\left(\mathrm{W} /{ }^{\wedge} 2\right) / 2$, where $\mathrm{V}$ is the tumor volume, $\mathrm{W}$ is the tumor width, and $\mathrm{L}$ is the tumor length. $\mathrm{RT}$ was performed using a $225 \mathrm{kVp}$ cabinet $\mathrm{x}$-ray irradiator filtered with 0.5 mm Cu (IC-250, Kimtron Inc.), and anesthetized animals were shielded with a $3.2-\mathrm{mm}$ lead shield with a 15 - to $20-\mathrm{mm}$ aperture. Radiation was administered in 6 fractionated doses of $2.5 \mathrm{~Gy}$ each, for a total dose of 15 Gy. Anti-PD1 and anti-Gal1 antibodies (Bristol-Myers Squibb) were injected (200 $\mu \mathrm{g}$ i.p.) every 4 days for a total of 5 doses, starting 1 day before RT. Control mice received rat IgG2a isotype antibodies. For orthotopic irradiation experiments, the PXi X-RAD SmART system was used to deliver the radiation focused on the buccal cavity.

In vitro transendothelial migration assays. Murine EC lines (C166 and SVEC) were cultured with MOC2 Gal1 WT CM and MOC2 Gal1-KO CM cells for 24 hours or with rGal1 protein (catalog 1245-GA-050, R\&D Systems). Next, the ECs were trypsinized, washed, and seeded (5000 live cells each) on the upper surface of a Transwell insert that had been previously coated with $0.2 \%$ (wt/vol) gelatin. ECs were cultured for 24 hours to form a compact monolayer, which was verified by visual inspection using an inverted microscope. Equal numbers of activated splenic $\mathrm{T}$ cells $\left(2 \times 10^{5}\right.$ to $\left.3 \times 10^{5}\right)$ were added to the upper chamber of each Transwell. The lower chamber was filled with RPMI plus 10\% FCS, along with CCL19 (500 ng/mL, catalog 587804, BioLegend) and CXCL10 (250 ng/mL, catalog 573604, BioLegend). After a 4-hour incubation at $37^{\circ} \mathrm{C}$, media in the lower chamber were collected, and the number of cells that migrated were counted with a hemocytometer. Triplicate wells were used for each treatment. The experiment was repeated at least 3 times. Transendothelial migration assays using isolated and expanded TECs from Gal1 WT and Gal1-KO tumors were performed at passage 4. For blocking experiments, the CM was preadsorbed with anti-Gal1 antibody, and then the CM was added to the ECs. For PD-L1 blockade, after treatment with $\mathrm{CM}$, the ECs were treated with a PD-L1-blocking antibody (catalog 124301, clone 10F.9G2, BioLegend) for 1 hour, after which the media were washed and T cells layered over ECs.

In vivo $T$ cell trafficking using adoptively transferred $T$ cells. Splenic T cells were isolated from MOC2 Gal1 WT or Gal1-KO tumor-bearing mice, after which the cells were labeled with $10 \mu \mathrm{M}$ CSFE (catalog 65-0850-84, Thermo Fisher Scientific) and transferred intravenously into (WT and KO) tumor-bearing mice (mean tumor size $75-100 \mathrm{~mm}^{3}$ ). Forty-eight hours after transfer, the mice were intravenously injected with rhodamine-dextran $(800 \mu \mathrm{g} / \mathrm{mouse}$, catalog D1824, Thermo Fisher Scientific), which labels the blood vessels, and Hoechst 33258 (250 $\mu \mathrm{g} /$ mouse, catalog H3569, Thermo Fisher Scientific), which labels the live cells. Ten minutes after injection, the mice were euthanized, and the number of $\mathrm{CSFE}^{+} \mathrm{CD}^{+} \mathrm{T}$ cells in the tumors and spleens of recipient mice were quantified using flow cytometry, and cryofixed sections of tissues were visualized using fluorescence microscopy.

Flow cytometric analyses. Following in vivo treatment, fresh tumor tissue was minced into 1-mm pieces and digested into a single suspension using the murine tumor dissociation kit (catalog 130-096-730, Miltenyi Biotec) according to the manufacturer's protocol. For peripheral blood analyses, $200 \mu \mathrm{l}$ blood was withdrawn via orbital bleeding, and plasma was collected. After RBC lysis, cells were resuspended in PBS, counted, and then stained with Zombie Aqua/Zombie Red (catalog 423109, BioLegend) for live/dead cell discrimination. Nonspecific binding was blocked using an anti-mouse CD16/CD32 antibody (catalog 101319, BioLegend). The cells were then processed for cell-surface staining, which was performed using the following antibodies from BioLegend: fluorophore-conjugated anti-mouse CD45.1 (30-F11), CD3 (17A2), TCR- $\beta$ (H57-597), CD4 (GK1.5), CD8 (53-6.7), PD1 (29F.1A12), Tim-3 (RMT3-23), NK1.1 (PK136), CD11b (M1/70), CD11c (N418), MHCII (M5/114.15.2), NFATc1 (7A6), and PD-L1 (10 F.9G2). After staining, the cells were washed and fixed in $4 \%$ paraformaldehyde for 20 minutes and then stored at $4^{\circ} \mathrm{C}$ overnight. Cell acquisition was performed the following day with FACSDiva software on an LSR II flow cytometer (BD Biosciences) and analyzed with FlowJo software (Tree Star). 
Isolation of ECs from mouse tissues. Mouse tumors and lungs were dissociated, and TECs and NECs were isolated using a magnetic cell-sorting system (catalog 130-096-730, Miltenyi Biotec) with antiCD31 microbeads (catalog 130-097-418, Miltenyi Biotec). CD45 depletion (catalog 130-052-301, Miltenyi Biotec) was carried out prior to CD31 labeling. Positive cells were sorted and plated on $0.1 \%$ gelatin-coated culture plates and cultured in endothelial growth medium from Cell Biologics. After a few days in culture and expansion, the CD $31^{+}$TECs were re-sorted to obtain enriched populations and then subcultured. RNA isolation and transendothelial migration assays were performed between passages 3 and 4. Expression analyses and sorting of TECs directly without passaging of cells was done at the Shared Stanford FACS facility.

Immunohistochemistry. Following excision from mice, tissues were fixed in $10 \% \mathrm{NBF}$ overnight at room temperature. For cryofixations, the tissues were fixed in 10\% NBF for 1 hour, embedded in O.C.T. Compound (catalog 25608-930, Tissue Tek), and then frozen on dry ice. Tissue blocks were sectioned at $5-\mu \mathrm{m}$ thickness on a microtome, placed onto positively charged glass slides, and stained with $\mathrm{H} \& \mathrm{E}$ according to standard protocols. For antibody staining of paraffin-embedded tissues, sections were deparaffinized and incubated with the respective primary antibodies (1:50 to 1:250) overnight at $4^{\circ} \mathrm{C}$, stained with a fluorescence-conjugated secondary antibody (1:500) 1 hour at room temperature, and finally mounted with DAPI (Vector Laboratories). Human Gal1 antibody was obtained from Gal1 antibody (BosterBio). Mouse and human PD-L1 antibodies (catalog ab233482) and human CD31 antibody (ab28364) were obtained from Abcam. Mouse CD31 antibody (clone SZ31, catalog DIA-310) was obtained from Dianova $\mathrm{GmbH}$. Human CD8 antibody (clone C8/144B, catalog M7103) was obtained from Dako. Immunofluorescence images were acquired with a Leica microscope, using $\times 40 / 0.95$ NA and $\times 63 / 1.4$ NA plan objectives.

Isolation of $T$ cells from spleens. Spleens from tumor-bearing mice were harvested under sterile conditions. Using mechanical disruption, the spleen (in PBS plus 2\% FCS) was dissociated into a cell suspension, which was then filtered through a $70-\mu \mathrm{m}$ filter to obtain a single-cell suspension. Following RBC lysis with ammonium chloride potassium (ACK) lysis buffer, T cells were isolated using a mouse Pan T cell Isolation Kit (catalog 19851, STEMCELL Technologies).

In vitro $T$ cell apoptosis. Molt- 4 cells $\left(2 \times 10^{5}\right)$, obtained from the American Type Culture Colelction (ATCC), were grown in RPMI 1640 medium with $10 \%$ FBS (200- $\mu$ l final volume) and incubated with $5 \mu \mathrm{M}$ human Gal1 (catalog 1245-GA-050, R\&D Systems) or 10 $\mu \mathrm{M}$ mouse Gal1 (1245-GA-050, R\&D Systems), either alone or in the presence of various concentrations $(0.2-20 \mu \mathrm{M})$ of anti-Gal1 antibody or mouse IgG1 isotype control antibody at $37^{\circ} \mathrm{C}$ for 5 hours. For inhibition of Gal1-induced T cell apoptosis, $1 \times \mathrm{PBS}$ and $0.1 \mathrm{M}$ lactose were used as negative and positive controls, respectively. After washing the cells twice with ice-cold PBS, cells were immediately analyzed by flow cytometry for viable and apoptotic cells using the Apoptosis Detection Kit (catalog 640914, BioLegend), following the manufacturer's protocol. The assay was also performed using activated mouse T cells treated with different concentrations of rGal1.

Cell agglutination assay. HL-60 cells $\left(5 \times 10^{-6}\right)$ grown in RPMI 1640 medium with $10 \% \mathrm{FBS}$ ( $50 \mu \mathrm{L}$ final volume) in a 96-well flat-bottomed plate were incubated at $37^{\circ} \mathrm{C}$ for 30 minutes with $2 \mu \mathrm{M}$ human Gal1, either alone or in the presence of $10 \mu \mathrm{M}$ anti-Gal1 antibody for testing the inhibition of Gal1-induced cell agglutination. $1 \times$ PBS was used as a negative control. Cells were examined under the microscope immediately after incubation and photographed.

TUNEL staining. Deparaffinized tumor sections were processed, labeled with the DeadEnd Fluorometric TUNEL System (cata$\log$ G3250, Promega), and mounted using VECTASHIELD DAPI (H-1200, Vector Laboratories).

Western blot analyses. For protein analysis, cells were grown to $70 \%$ confluence, washed with PBS, and then lysed using $150 \mu \mathrm{l}$ RIPA lysis buffer (50 mM Tris- $\mathrm{HCl}$ [pH 7.4], $150 \mathrm{mM} \mathrm{NaCl}, 1 \% \mathrm{NP}-40,0.1 \%$ SDS, $0.5 \%$ sodium deoxycholate) supplemented with the Halt Protease and Phosphatase Inhibitor Cocktail (catalog 78440, Pierce, Thermo Fisher Scientific) and incubated on ice for 20 minutes. Cells were scraped and collected, followed by centrifugation at $16,000 \mathrm{~g}$ for 10 minutes at $4^{\circ} \mathrm{C}$. The supernatant was collected and protein estimation was performed using a BCA Protein Assay kit (catalog 23225, Pierce, Thermo Fisher Scientific). Lysate (30-60 $\mu \mathrm{g})$ was then mixed with $4 \times$ SDS sample buffer (40\% glycerol, $8 \%$ SDS, $0.24 \mathrm{M}$ Tris- $\mathrm{HCl}$, and $10 \%$ 2-mercaptoethanol). Samples were heated at $95^{\circ} \mathrm{C}$ for 5 minutes and then resolved by SDS-PAGE according to standard protocols. The following primary antibodies were used to detect specific proteins: Gal1 (1:1000; catalog AF1245 [mouse], catalog AF1152, R\&D Systems), pSTAT1 (1:1000; catalog 7647, Cell Signaling Technology), STAT1 (1:1000; catalog 9172, Cell Signaling Technology), pJAK (1:1000; catalog 3771, Cell Signaling Technology), total JAK2 (1:1000; cata$\log 3230$, Cell Signaling Technology), and $\beta$-actin (1:5000; sc-47778 HRP, Santa Cruz Biotechnology). The following secondary antibodies were used in this study: HRP-conjugated goat anti-mouse (1:10,000; Zymed Laboratories) and HRP-conjugated goat anti-rabbit (1:5000; Cell Signaling Technology). Immunoblots were developed with Pierce West Pico (catalog 35060, Thermo Fisher Scientific) and visualized using the ChemiDoc XRS imaging system equipped with Image Lab Software (Bio-Rad Laboratories). (See the complete unedited blots in the supplemental material.)

Real-time PCR. RNA was isolated using TRIzol (Invitrogen, Thermo Fisher Scientific) and treated with DNase I (catalog EN0525, Thermo Fisher Scientific). First-strand cDNA synthesis was performed with a SuperScript II Reverse Transcriptase Kit (catalog 18064022, Thermo Fisher Scientific) according to the manufacturer's protocol. Quantitative real-time PCR was carried out using Power SYBR Green Master Mix (catalog 4367659, Thermo Fisher Scientific). The reaction was run and analyzed using the ABI-7900HT Fast Real-Time PCR System (Applied Biosystems), with normalization to mouse $\beta$-actin. The mouse primer sequences used for amplification are listed in Supplemental Table 1.

TCGA data analyses. RNA-Seq data from the TCGA - Head and Neck Squamous Cell Carcinoma (HNSC) cohort were downloaded through the data hub within the University of California Santa Cruz (UCSC) Xena resource (https://xena.ucsc.edu/). The gene expression profile was measured experimentally using the Illumina HiSeq 2000 RNA Sequencing platform at the University of North Carolina TCGA genome characterization center. Level 3 data were downloaded from the TCGA data coordination center. This data set shows the gene-level transcription estimates, as in the $\log _{2}(x+1)$ transformed RSEM normalized count. Genes were mapped onto the human genome coordinates using the UCSC Xena HUGO probeMap. The RNA-Seq expression data were then processed through CIBERSORT (https://cibersort.stanford.edu/) (40) using the default LM22 immune cell gene signatures and 100 permutations, with the 
quantile normalization function disabled. Only Relative Mode was used, by which CIBERSORT estimates the relative fraction of each cell type in the signature matrix, such that the sum of all fractions is equal to 1 for a given mixture sample. In addition, HNC TCGA data sets from 518 patients in the Gene Expression Profiling Interactive Analysis (GEPIA) database (http://cancergenome.nih.gov) were used to explore the relationship between LGALS1 expression and survival of patients with HNC. Kaplan-Meier survival plots were obtained using the GEPIA online tool. The GEPIA 2 online tool (http://gepia2. cancer-pku.cn/\#analysis) was used to produce a box plot of LGALS1, LGALS3, and LGALS9 transcriptional expression in 518 patients with HNC (TCGA cohort) and 44 normal samples (GTEx cohort). The $P$ value cutoff was set at 0.01 . The result was normalized and $\log _{2}$ scaled using the GEPIA online analysis tool.

Statistics. Flow cytometric analyses and other in vitro assays were analyzed using an unpaired, 2-tailed Student's $t$ test, and 1-way ANOVA models with post hoc comparisons were performed using Tukey's or Dunnett's adjustment as appropriate. Growth curves were analyzed in a repeated-measures model in order to account for the within-mouse correlation. All data are presented as the mean \pm SD, and a $P$ value of 0.05 or less was considered statistically significant. Timeto-event outcomes such as time to overall survival were summarized with Kaplan-Meier curves, and groups were compared using log-rank tests. The rates of response to immunotherapy and the distribution of galectin staining were analyzed with a $\chi^{2}$ test. All analyses were performed in SAS, version 9.4 (SAS Institute) or GraphPad Prism, version 7.04 (GraphPad Software).

Study approval. All the animal studies were conducted in compliance with the Administrative Panel on Laboratory Animal Care (APLAC) guidelines and approved by the IACUC of Stanford University, under APLAC protocol number 15106 (Research Compliance
Office, Palo Alto, California, USA). Collection of patient data was approved by the Stanford Institutional Review Board (IRB no. 40425). Written informed consent was received from all participants prior to their inclusion in this study. Details on the patients with HNSCC included in this study are provided in Table 1 .

\section{Author contributions}

DKN and QTL conceived the project. DKN, TA, and QL designed, implemented experiments, and analyzed data. HC assisted with animal experiments. ADC and SA assisted with collection of samples from patients. SK and CK assisted with patient sample processing, staining, and scoring. JB assisted with ELISA studies. AK, VSR, and ZW assisted with Gal1 antibody development. RU provided the HPV murine oral cancer cells MOC1 and MOC2 as well as reagents and edited the manuscript. RVE and DJ assisted with data interpretation and statistical analysis. ACK and AG helped with conceptual study design and edited the manuscript. RL helped with clinical sample collection and analyses and IRB approval. DKN and QTL wrote the manuscript.

\section{Acknowledgments}

We thank William Spanos for the $\mathrm{HPV}^{+}$cell lines. We acknowledge support form the NIH (R01CA161585-03 and P01CA067166). We thank Colleen and Rick Pouliot for their support of the project. We also acknowledge Manish Chamoli for the exchange of ideas and provision of reagents for the project.

Address correspondence to: Quynh Thu Le, Department of Radiation Oncology - Radiation Therapy, Stanford University School of Medicine, 875 Blake Wilbur Drive, Stanford, California 94305, USA. Phone: 650.725.0203; Email: qle@stanford.edu.
1. Postow MA, Callahan MK, Wolchok JD. Immune Checkpoint Blockade in Cancer Therapy. JClin Oncol. 2015;33(17):1974-1982.

2. Ferris RL, Blumenschein G Jr, Fayette J, Guigay J, Colevas AD, Licitra L, et al. Nivolumab for recurrent squamous-cell carcinoma of the head and neck. N Engl JMed. 2016;375(19):1856-1867.

3. Galon J, et al. Type, density, and location of immune cells within human colorectal tumors predict clinical outcome. Science. 2006;313(5795):1960-1964.

4. Hwang WT, Adams SF, Tahirovic E, Hagemann IS, Coukos G. Prognostic significance of tumor-infiltrating T cells in ovarian cancer: a meta-analysis. Gynecol Oncol. 2012;124(2):192-198.

5. Herbst RS, et al. Predictive correlates of response to the anti-PD-L1 antibody MPDL3280A in cancer patients. Nature. 2014;515(7528):563-567.

6. Joyce JA, Fearon DT. T cell exclusion, immune privilege, and the tumor microenvironment. Science. 2015;348(6230):74-80.

7. Chen PL, et al. Analysis of Immune Signatures in Longitudinal Tumor Samples Yields Insight into Biomarkers of Response and Mechanisms of Resistance to Immune Checkpoint Blockade. Cancer Discov. 2016;6(8):827-837.

8. Mauge L, Terme M, Tartour E, Helley D. Control of the adaptive immune response by tumor vasculature. Front Oncol. 2014;4:61.
9. Schmittnaegel M, De Palma M. Reprogramming tumor blood vessels for enhancing immunotherapy. Trends Cancer. 2017;3(12):809-812.

10. Motz GT, et al. Tumor endothelium FasL establishes a selective immune barrier promoting tolerance in tumors. Nat Med. 2014;20(6):607-615.

11. Lanitis E, Irving M, Coukos G. Targeting the tumor vasculature to enhance $\mathrm{T}$ cell activity. Curr Opin Immunol. 2015;33:55-63.

12. Demaria O, et al. STING activation of tumor endothelial cells initiates spontaneous and therapeutic antitumor immunity. Proc Natl Acad Sci USA. 2015;112(50):15408-15413.

13. Cousin JM, Cloninger MJ. The role of galectin-1 in cancer progression, and synthetic multivalent systems for the study of galectin-1. Int JMol Sci. 2016;17(9):E1566.

14. Le QT, et al. Galectin-1: a link between tumor hypoxia and tumor immune privilege. J Clin Oncol. 2005;23(35):8932-8941.

15. Saussez S, Camby I, Toubeau G, Kiss R. Galectins as modulators of tumor progression in head and neck squamous cell carcinomas. Head Neck. 2007;29(9):874-884.

16. Stanley P. Galectin-1 pulls the strings on VEGFR2. Cell. 2014;156(4):625-626.

17. Rabinovich GA, Conejo-García JR. Shaping the immune landscape in cancer by galectin-driven regulatory pathways. J Mol Biol.
2016;428(16):3266-3281

18. Facciabene A, et al. Local endothelial complement activation reverses endothelial quiescence, enabling t-cell homing, and tumor control during t-cell immunotherapy. Oncoimmunology. 2017;6(9):e1326442.

19. Cedeno-Laurent F, Dimitroff CJ. Galectin-1 research in T cell immunity: past, present and future. Clin Immunol. 2012;142(2):107-116.

20. Stowell SR, et al. Differential roles of galectin-1 and galectin-3 in regulating leukocyte viability and cytokine secretion. JImmunol. 2008;180(5):3091-3102.

21. Büchel G, et al. Immune response modulation by galectin-1 in a transgenic model of neuroblastoma. Oncoimmunology. 2016;5(5):e1131378.

22. Judd NP, Allen CT, Winkler AE, Uppaluri R. Comparative analysis of tumor-infiltrating lymphocytes in a syngeneic mouse model of oral cancer. Otolaryngol Head Neck Surg. 2012;147(3):493-500.

23. Vermeer DW, et al. Metastatic model of $\mathrm{HPV}^{+}$ oropharyngeal squamous cell carcinoma demonstrates heterogeneity in tumor metastasis. Oncotarget. 2016;7(17):24194-24207.

24. Saussez S, et al. The determination of the levels of circulating galectin-1 and - 3 in HNSCC patients could be used to monitor tumor progression and/or responses to therapy. Oral Oncol. 2008;44(1):86-93. 
25. Perillo NL, Pace KE, Seilhamer JJ, Baum LG. Apoptosis of T cells mediated by galectin-1. Nature. 1995;378(6558):736-739.

26. Kovács-Sólyom F, et al. Mechanism of tumor cell-induced T-cell apoptosis mediated by galectin-1. Immunol Lett. 2010;127(2):108-118.

27. Dias-Baruffi M, Zhu H, Cho M, Karmakar S, McEver RP, Cummings RD. Dimeric galectin-1 induces surface exposure of phosphatidylserine and phagocytic recognition of leukocytes without inducing apoptosis. J Biol Chem. 2003;278(42):41282-41293.

28. Stowell SR, Karmakar S, Stowell CJ, Dias-Baruffi M, McEver RP, Cummings RD. Human galectin-1, -2 , and -4 induce surface exposure of phosphatidylserine in activated human neutrophils but not in activated T cells. Blood. 2007;109(1):219-227.

29. Thijssen VL, Griffioen AW. Galectin-1 and -9 in angiogenesis: a sweet couple. Glycobiology. 2014;24(10):915-920.

30. Weis SM, Cheresh DA. Tumor angiogenesis: molecular pathways and therapeutic targets. Nat Med. 2011;17(11):1359-1370.

31. Kuo P, et al. Galectin-1 mediates radiation-related lymphopenia and attenuates NSCLC radiation response. Clin Cancer Res. 2014;20(21):5558-5569.

32. Dovedi SJ, et al. Acquired resistance to fractionated radiotherapy can be overcome by concurrent PD-L1 blockade. Cancer Res. 2014;74(19):5458-5468.

33. Motz GT, Coukos G. The parallel lives of angiogenesis and immunosuppression: cancer and other tales. Nat Rev Immunol. 2011;11(10):702-711.

34. Tian L, et al. Mutual regulation of tumour vessel normalization and immunostimulatory reprogramming. Nature. 2017;544(7649):250-254.

35. Rodig N, et al. Endothelial expression of PD-L1 and PD-L2 down-regulates CD8+ T cell activation and cytolysis. Eur J Immunol. 2003;33(11):3117-3126.

36. Blouin CM, et al. Glycosylation-dependent IFN- $\gamma \mathrm{R}$ partitioning in lipid and actin nanodomains is critical for JAK activation. Cell. 2016;166(4):920-934.

37. Benci JL, et al. Tumor interferon signaling regulates a multigenic resistance program to immune checkpoint blockade. Cell. 2016;167(6):1540-1554.e12.

38. Croci DO, et al. Glycosylation-dependent lectin-receptor interactions preserve angiogenesis in anti-VEGF refractory tumors. Cell. 2014;156(4):744-758.

39. Wu X, et al. Combined anti-VEGF and anti-CTLA-4 therapy elicits humoral immunity to galectin-1 which is associated with favorable clinical outcomes. Cancer Immunol Res. 2017;5(6):446-454.

40. Newman AM, et al. Robust enumeration of cell subsets from tissue expression profiles. Nat Methods. 2015;12(5):453-457. 